\title{
Wannier-function description of the electronic polarization and infrared absorption of high-pressure hydrogen
}

\author{
Ivo Souza ${ }^{1}$, Richard M. Martin ${ }^{1}$, Nicola Marzari ${ }^{2}$, Xinyuan Zhao ${ }^{3}$, and David Vanderbilt ${ }^{3}$ \\ ${ }^{1}$ Department of Physics and Materials Research Laboratory, University of Illinois, Urbana, IL 61801 \\ ${ }^{2}$ Department of Chemistry, Princeton University, Princeton, NJ 08544-1009 \\ 3 Department of Physics and Astronomy, Rutgers University, Piscataway, NJ 08854-0849
}

(November 17, 2018)

\begin{abstract}
We have constructed maximally-localized Wannier functions for prototype structures of solid molecular hydrogen under pressure, starting from LDA and tight-binding Bloch wave functions. Each occupied Wannier function can be associated with two paired protons, defining a "Wannier molecule". The sum of the dipole moments of these "molecules" always gives the correct macroscopic polarization, even under strong compression, when the overlap between nearby Wannier functions becomes significant. We find that at megabar pressures the contributions to the dipoles arising from the overlapping tails of the Wannier functions is very large. The strong vibron infrared absorption experimentally observed in phase III, above $\sim 150 \mathrm{GPa}$, is analyzed in terms of the vibron-induced fluctuations of the Wannier dipoles. We decompose these fluctuations into "static" and "dynamical" contributions, and find that at such high densities the latter term, which increases much more steeply with pressure, is dominant.
\end{abstract}

PACS numbers: $62.50+\mathrm{p}, 78.30 .-\mathrm{j}, 64.30 .+\mathrm{t}, 71.15 . \mathrm{Mb}$

\section{INTRODUCTION}

\section{A. Wannier functions}

The electronic structure of periodic solids is usually described, in the independent-electron approximation, in terms of the extended Bloch eigenfunctions. An alternative representation is provided by the Wannier functions (WFs) [迎], which are localized, with a typical spread of the order of the atomic dimensions; they can be obtained via a unitary transformation of the Bloch states belonging to an isolated band [1] or to a composite group of bands [2,3] (i.e., bands that may be connected among themselves by degeneracies, but are separated from all others by energy gaps). For some purposes the latter description is advantageous: for instance, the WFs constructed from the states in the valence bands provide an intuitive, "chemical-like" localized picture of bonding and dielectric properties of insulators [3].

The major drawback of the Wannier representation is the strong nonuniqueness of the WFs: their average location, shape, and spread all depend on the arbitrary choice of gauge [2.33]. In practice, this indeterminacy can be resolved by working with the set of WFs which is most localized according to some sensible criterion. A certain degree of arbitrariness still remains regarding which measure of localization to use, and in fact several alternatives have been proposed in the literature. We follow the approach of Ref. [3], which amounts to minimizing the sum of the quadratic spreads of the WFs (see Sec. III). We will use density-functional theory in the local density approximation (LDA), complemented by a tight-binding analysis, to investigate in terms of well-localized WFs the elec- tronic structure and dielectric properties of compressed molecular hydrogen [4].

\section{B. Compressed molecular hydrogen}

Solid hydrogen under pressure has attracted considerable attention over the years [5, and the main goal has been to try to metallize it; this is expected to occur at high enough pressures, either by band gap closure in a molecular phase, or by molecular dissociation, whichever occurs first [6.7]. However, up to the highest pressures reached so far $(\sim 340 \mathrm{GPa})$, hydrogen appears to remain both molecular and insulating [8]. Nevertheless, a rich phase diagram has emerged, with three distinct phases unambiguously identified using Raman and infrared (IR) spectroscopy [5].

The precise crystal structure of the high-pressure phases (phases II and III) has not been determined experimentally, and conclusive theoretical predictions have proven quite difficult, due to the quantum effects associated with the protons. The purpose of the present work is not to propose new candidate structures, but rather to make some very general points, illustrated on a couple of particularly simple prototype structures (Fig. 11), which were chosen mainly for clarity. It is hoped that, even if none of them turns out to be the correct structure of phase III (which is likely to be the case), they manage to capture some of its relevant features. For instance, ab-initio calculations at megabar pressures and low temperatures tend to favor structures in which the centers of the molecules form an hcp or, more generally, a triangular lattice [9.10] (possibly with a small distortion [11]) [12]. It is believed that in phase III the molecules are ori- 

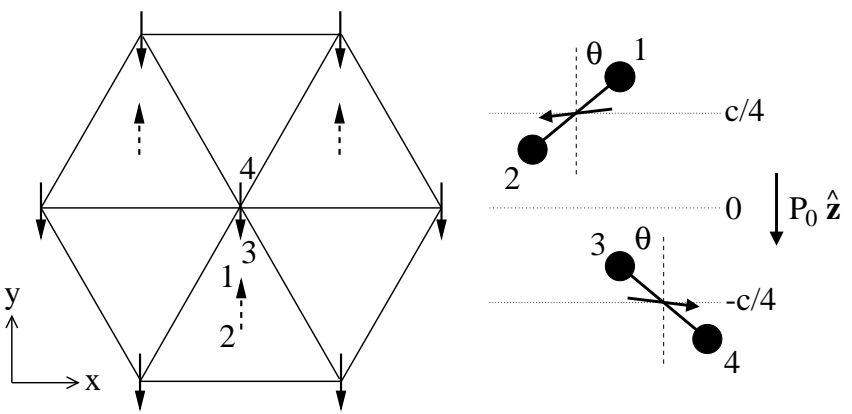

FIG. 1. The $C m c 2_{1}$ structure viewed along the c-axis (left) and in the $y z$ plane (right). The centers of the molecules lie on hcp sites, and the molecules in the two sublattices are tilted away from the c-axis by opposite angles $\theta$ and $-\theta$. The $C 2 / m$ structure is identical except that the two molecules in the primitive cell are tilted in the same direction by an angle $\theta$. The arrows on the right side indicate the directions of the dipoles of the two "Wannier molecules" for $r_{s}=1.52$.

entationally ordered, with their axes tilted away from the $c$-axis, as such canted structures tend to be more stable [7,9,10,13]. Moreover, the resulting lowering of symmetry gives rise to IR-active vibron modes [14, 15]; indeed, one of the signatures of phase III, above $150 \mathrm{GPa}$, is a strong IR absorption peak in the vibron frequency range [16, 17], which contrasts with the much weaker absorption found in phases I and II.

\section{Organization}

The paper is organized as follows. In Sec. II we briefly review the method used for constructing well-localized WFs; in Sec. III we investigate the permanent dipole moments of the "Wannier molecules" as a function of crystal structure and pressure; the results are presented in terms of a "static effective charge" vector associated with each molecule. In Sec. IV we look at the vibroninduced fluctuations of those dipoles, which can be quantified in terms of a "vibron-induced effective charge" vector on each molecule. From the effective charges the strength of the vibron IR absorption is calculated and compared with experiment, and the relative importance of the "static" and "dynamical" charge contributions is ascertained. Sec. V deals with the spatial distribution of the WFs and the effect of molecular overlap on the dielectric properties of the compressed solid. In Sec. VI we investigate the nonuniqueness associated with the definition of well-localized WFs in the dense solid. The tightbinding analysis is presented in Sec. VII. In Sec. VIII we give a discussion of our results.

Atomic units are used for all quantities except pressures, which are in gigapascal $(\mathrm{GPa})$, and energies, in electron-volt $(\mathrm{eV})$. Densities are expressed in terms of $r_{s}$, defined as $\left(4 \pi r_{s}^{3} / 3\right) a_{0}^{3}=V / N$, where $N$ is the number of protons in the volume $V$, and $a_{0}$ is the Bohr radius. Since the LDA tends to underestimate the pres- sures, in order to convert from $r_{s}$ to pressure we use an experimental equation of state extrapolated to high pressures [18]. The LDA calculations were performed using a plane-wave cutoff of $90 \mathrm{Ry}$ and the bare Coulomb potential of the protons. The self-consistent calculations with 4 atoms per cell used a $(11,11,11)$ MonkhorstPack mesh for the Brillouin zone sampling. After selfconsistency was achieved, the "maxloc" WFs were determined starting from the Bloch states again calculated on a $(11,11,11)$ mesh. In all the calculations we have used the following parameters for the $C m c 2_{1}$ structure described in Fig. 1: $r_{\text {bond }}=1.445$ a.u., $c / a=1.576$, and the tilt angle $\theta=54.0^{\circ}$. For $C 2 / \mathrm{m}$ the parameters are $r_{\text {bond }}=1.456$ a.u., $c / a=1.588$, and $\theta=69.5^{\circ}$. In both cases the structures were obtained by minimizing the enthalpy at a fixed LDA pressure of $100 \mathrm{GPa}$, with a resulting density of $r_{s}=1.52$ (which experimentally corresponds to about $115 \mathrm{GPa}$, according to Ref. [18]). The same parameters were used at all other densities.

\section{MAXIMALLY-LOCALIZED WANNIER FUNCTIONS}

A set of WFs $\left\{w_{n}(\mathbf{r}-\mathbf{R})\right\}$, each labeled by a different Bravais lattice vector $\mathbf{R}$, can be constructed from the Bloch eigenstates $\left\{\psi_{n \mathbf{k}}\right\}$ in band $n$ using the unitary transformation

$$
w_{n}(\mathbf{r}-\mathbf{R})=\frac{v}{8 \pi^{3}} \int_{\mathrm{BZ}} e^{-i \mathbf{k} \cdot \mathbf{R}} \psi_{n \mathbf{k}} d \mathbf{k},
$$

where $v$ is the volume of the unit cell of the crystal and the integral is over the Brillouin zone. Except for the constraint $\psi_{n, \mathbf{k}+\mathbf{G}}=\psi_{n \mathbf{k}}$ for all reciprocal lattice vectors $\mathbf{G}$, the overall phases of the Bloch functions $\psi_{n \mathbf{k}}=e^{i \mathbf{k} \cdot \mathbf{r}} u_{n \mathbf{k}}$ are at our disposal. However, a different choice of phases (or "gauge"),

$$
u_{n \mathbf{k}} \rightarrow e^{i \varphi_{n}(\mathbf{k})} u_{n \mathbf{k}}
$$

does not translate into a simple change of the overall phases of the WFs; their shape and spatial extent will in general be affected, while the location of their centers of charge remains invariant modulo a lattice vector $\mathbf{R}$ [3]. If the band is isolated, Eq. 2 is the only allowed type of gauge transformation for changing the WF $w_{n}(\mathbf{r})$ associated with that band. In the case of a composite group of bands, the allowed transformations are of the more general form

$$
u_{n \mathbf{k}} \rightarrow \sum_{m} U_{m n}^{(\mathbf{k})} u_{m \mathbf{k}}
$$

where $U_{m n}^{(\mathbf{k})}$ is a unitary matrix that mixes the bands at every wave vector $\mathbf{k}$. Under this transformation the individual Wannier centers can shift, but their sum over the group of bands is preserved modulo a lattice vector [3]. Once a measure of localization has been chosen and the 
group of bands specified, the search for the corresponding set of "maximally-localized" WFs becomes a problem of functional minimization in the space of the matrices $U_{m n}^{(\mathbf{k})}$. The strategy of Ref. [3] is to minimize the sum of the quadratic spreads of the Wannier probability distributions $\left\{\left|w_{n}(\mathbf{r})\right|^{2}\right\}$, given by

$$
\Omega=\sum_{n}\left(\left\langle r^{2}\right\rangle_{n}-\langle\mathbf{r}\rangle_{n}^{2}\right)
$$

where the sum is over the chosen group of bands (in our application they will be the valence bands), and $\langle\mathbf{r}\rangle_{n}=\int \mathbf{r}\left|w_{n}(\mathbf{r})\right|^{2} d \mathbf{r}$, etc. Interestingly, the resulting "maximally-localized" (or "maxloc") WFs turn out to be real, apart from an arbitrary overall phase [3].

In numerical calculations the Bloch states $\psi_{n \mathbf{k}}$ are computed on a regular mesh of $k$-points in the Brillouin zone; the integral in Eq. 1 is then replaced by a sum over the points in the mesh. In Ref. [3] an expression was derived for the gradient of the spread functional $\Omega$ with respect to an infinitesimal rotation $\delta U_{m n}^{(\mathbf{k})}$ of the set of Bloch orbitals, in terms of the Bloch functions in such a mesh. The only information needed for calculating the gradient are the overlaps $\left\langle u_{m \mathbf{k}} \mid u_{n, \mathbf{k}+\mathbf{b}}\right\rangle$, where $\mathbf{b}$ are vectors connecting each mesh point to its near neighbors. Once the gradient is computed, the minimization can then proceed via a steepest-descent or conjugate-gradient algorithm.

Since the Bloch eigenstates at different $k$-points are initially computed by independent numerical matrix diagonalizations, their phases are unrelated. As a consequence, the WFs obtained directly from them using the discretized version of Eq. 1 will be poorly localized, or not localized at all. In practice the following strategy is used for preparing a better set of Bloch states as the starting point for the minimization: one chooses a set of localized "trial functions" in the unit cell, which constitute a rough initial guess at the WFs; for solid hydrogen we use Gaussians on the centers of the molecules. Then, a unitary rotation among the initial Bloch orbitals is made in order to maximize their projections onto these trial functions (the detailed procedure is described in Eqs. 6264 of Ref. [3]). For a reasonable choice of the width of the Gaussians (we have used a r.m.s. width of $1 \AA$ ), the resulting rotated WFs are already extremely close to the "maxloc" ones, as discussed in Secs. VI and VII A.

\section{EQUILIBRIUM WANNIER DIPOLES}

The neutral entity composed of two paired nuclei and the occupied "maxloc" Wannier orbital centered around them forms a "Wannier molecule" in the bulk of the solid. In the low-density limit the "maxloc" WFs become nonoverlapping and coincide with the ground state bonding orbitals of isolated $\mathrm{H}_{2}$ molecules; however, at the high pressures we are interested in, there is an appreciable overlap between neighboring WFs. In what follows

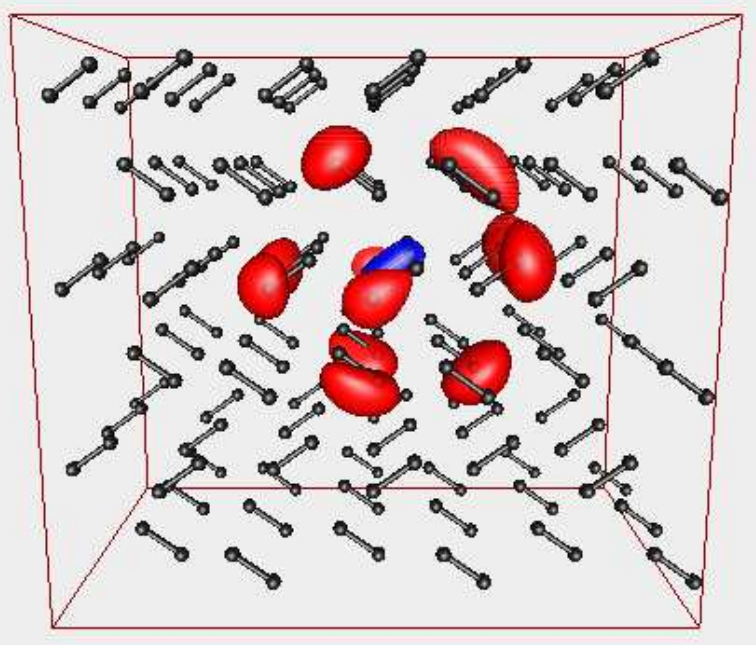

FIG. 2. Contour plot of $\sqrt{v} w_{1}(\mathbf{r})$ for an occupied Wannier function in the $C m c 2_{1}$ structure at $r_{s}=1.52(v=59.3$ a.u. is the volume of the primitive cell). The central, cylindrically-shaped contour, which represents the bonding part of the $\mathrm{WF}$, has a positive amplitude of +2.12 ; the outer lobes ("orthogonality tails"), with antibonding character, have an amplitude of -0.11 .

we will sometimes loosely refer to the "maxloc" Wannier molecules in the dense solid simply as "molecules". One should keep in mind, however, that had we chosen a different measure of localization, the resulting "maximallylocalized" WFs in the dense system would in general differ somewhat from the ones we obtain. This nonuniqueness is intrinsic to WFs, and can be viewed as a manifestation of the ambiguity that always arises when trying to define "molecules" in a dense medium, either in terms of WFs or by other means. These issues will be discussed in Secs. VI and VIIIB.

Fig. 22 shows a contour plot of a Wannier orbital for $C m c 2_{1}$ at $r_{s}=1.52$. The central positive contour with a large amplitude represents the molecular bond. The lowering of symmetry due to the crystalline environment is clear from the shape of the outer "corona" formed by the negative lobes, which have an antibonding character. These so-called "orthogonality tails" appear when the molecules overlap, due to the orthogonality requirement between different WFs; they are concentrated around the twelve nearest molecules, which allows for an efficient orthogonalization between neighboring WFs. We will argue in Sec. $\nabla$ that these overlapping orthogonality tails strongly influence the dielectric properties, and in particular the vibron IR activity.

An important effect of the anisotropic crystalline environment is that the molecules becomes polarized under the self-consistent internal electric fields inside the solid. In commonly used treatments of the dielectric properties of molecular crystals 19,20, the so-called Clausius- 
TABLE I. Static (Eq. 7) and vibron-induced (Eq. 8) effective charge vectors for the two molecules in the primitive cell of the $C m c 2_{1}$ structure. The $x$-components vanish by symmetry.

\begin{tabular}{ccccccc}
\hline \hline Molecule & \multicolumn{2}{c}{ Static } & \multicolumn{2}{c}{ In-phase } & \multicolumn{2}{c}{ Out-of-phase } \\
\hline 1 & $q_{y}^{\mathrm{s}}(1)$ & $q_{z}^{\mathrm{s}}(1)$ & $q_{y}^{\mathrm{i}}(1)$ & $q_{z}^{\mathrm{i}}(1)$ & $q_{y}^{\mathrm{o}}(1)$ & $q_{z}^{\mathrm{o}}(1)$ \\
2 & $-q_{y}^{\mathrm{s}}(1)$ & $q_{z}^{\mathrm{s}}(1)$ & $-q_{y}^{\mathrm{i}}(1)$ & $q_{z}^{\mathrm{i}}(1)$ & $q_{y}^{\mathrm{o}}(1)$ & $-q_{z}^{\mathrm{o}}(1)$ \\
\hline \hline
\end{tabular}

TABLE II. Same as Table [i, but for the $C 2 / m$ structure.

\begin{tabular}{ccccccc}
\hline \hline Molecule & \multicolumn{2}{c}{ Static } & \multicolumn{2}{c}{ In-phase } & \multicolumn{2}{c}{ Out-of-phase } \\
\hline 1 & $q_{y}^{\mathrm{s}}(1)$ & $q_{z}^{\mathrm{s}}(1)$ & $q_{y}^{\mathrm{i}}(1)$ & $q_{z}^{\mathrm{i}}(1)$ & $q_{y}^{\mathrm{o}}(1)$ & $q_{z}^{\mathrm{o}}(1)$ \\
2 & $-q_{y}^{\mathrm{s}}(1)$ & $-q_{z}^{\mathrm{s}}(1)$ & $-q_{y}^{\mathrm{i}}(1)$ & $-q_{z}^{\mathrm{i}}(1)$ & $q_{y}^{\mathrm{o}}(1)$ & $q_{z}^{\mathrm{o}}(1)$ \\
\hline \hline
\end{tabular}

Mossotti approximation is assumed: the system is modeled as a sum of nonoverlapping molecular charge distributions which become polarized in the local field produced by the surrounding molecules; the bulk polarization is then the sum of the individual, nonoverlapping, molecular dipole moments, which can be straightforwardly calculated from the bulk charge density $\rho(\mathbf{r})$. Such a description becomes inappropriate whenever there is significant molecular overlap [21]: the electron density becomes different from zero everywhere, and as a result the net dipole moment becomes dependent on the particular choice made for the unit cell [22,23]. In the case of molecular crystals, this is expected to occur, for instance, when the system is strongly compressed [24; under such circumstances a more careful treatment of the macroscopic polarization is required. According to the Berry phase theory of bulk polarization [23], whenever such overlap effects are significant, the macroscopic polarization $\mathbf{P}_{\text {mac }}$ of an insulating crystal cannot be extracted from the bulk $\rho(\mathbf{r})$, and is instead given, in the independent-electron approximation, by a Berry phase of the occupied Bloch states. This is a gauge-invariant quantity, and it is identical to another invariant, the total sum of the dipoles $\mathbf{d}(n)$ of the "Wannier molecules" [23]:

$$
\begin{gathered}
\mathbf{P}_{\mathrm{mac}}=\frac{1}{v} \sum_{n=1}^{M} \mathbf{d}(n), \\
\mathbf{d}(n)=-2 e \int \mathbf{r}\left|w_{n}(\mathbf{r})\right|^{2} d \mathbf{r},
\end{gathered}
$$

where $M$ is the number of valence bands and $e$ is the magnitude of the electron charge. In Eq. 6 the origin is chosen midway between the two paired protons, to cancel their contributions. The factor of 2 comes from spindegeneracy (each occupied WF carries two electrons). We stress that Eq. F does not rely on the ClausiusMossotti approximation at all, and it remains exact even when the "Wannier molecules" overlap strongly, as long as the system remains insulating. The decomposition of $\mathbf{P}_{\text {mac }}$ into individual Wannier dipoles is a powerful analysis tool, allowing us to go beyond the Berry phase approach used in Ref. [25], which only gives the net polarization of the unit cell.

The permanent Wannier dipoles can be used to assign to each molecular charge distribution a "static effective charge" vector:

$$
\mathbf{q}^{\mathrm{s}}(n)=\frac{\mathbf{d}(n)}{r_{\text {bond }}(n)},
$$

where $r_{\text {bond }}(n)$ is the equilibrium bond length. This quantity, which vanishes in the low density limit of isolated molecules, measures the spontaneous symmetrybreaking charge transfer which occurs in the compressed solid whenever the two atoms in a molecule occupy crystallographically inequivalent sites. We emphasize that this definition is somewhat arbitrary, for the reasons discussed at the beginning of this section (but see Sec. VI), and therefore $\mathbf{q}^{\mathrm{s}}(n)$ does not relate directly to any measurable quantity. However, it is a sensible definition, which reduces to the natural one in the extreme ionic limit where the electron distribution is strongly concentrated around the ions. In the next section we will use it to decompose the vibron effective charge into "static" and "dynamical" contributions, with the aim of understanding the origin of the strong IR absorption in phase III.

The location of the centers of the "maxloc" WFs reflects the symmetry properties of the crystal; this is apparent from the form of the vectors $\mathbf{q}^{\mathrm{s}}(n)$, shown in the first set of columns in Tables If and II for the two structures studied. The first set of columns in Table III lists their explicit values for $r_{s}=1.52$, both with the molecules on-site and after allowing them to move away from the ideal hcp sites [11,25]. For comparison we also report the values calculated using an electric quadrupolar (EQ) model [26]. In the $C 2 / m$ structure there is a center of inversion between the two molecules in the primitive cell, leading to a cancellation of their permanent dipoles; in the lower symmetry $C m c 2_{1}$ the $y$-components of the individual dipoles still average to zero over the primitive cell, but the $z$-components add up, yielding a small spontaneous polarization along the $c$ axis (see Fig. 1). In general $\left|q_{y}^{\mathrm{s}}\right|>>\left|q_{z}^{\mathrm{s}}\right|$, so that the dipole moments make an angle with the molecular axes, i.e., $q_{\perp}^{\mathrm{s}}$ in Table III is nonzero, although it is smaller than $q_{\|}^{\mathrm{s}}$. This agrees with the EQ model, where for the hcp-centered (on-site) structures the quadrupolar field at the center of the molecules is along $y$, so that the dipole moment along $z$ is solely due to the small anisotropy in the polarizability 25]. The EQ model also predicts larger dipoles in the $C 2 / m$ than in the $C m c 2_{1}$ structure (although the effect is not nearly as pronounced as in the LDA WFs), as well as an increase in their magnitude as the molecules move off-site [11,25. Although some of the qualitative features of the LDA Wannier dipoles are captured by the EQ model, its predictions are not reliable: for instance, it does not re- 
TABLE III. Static (Eq. 月) and vibron-induced (Eq. 8) effective charge vectors for molecule 1, for the hcp-centered (on-site) and the off-site structures, at $r_{s}=1.52$. Results are presented for the Wannier functions in the LDA approximation (WF) and for the electric quadrupole model (EQ). The $x$-components vanish by symmetry. $q_{\|}$and $q_{\perp}$ are the magnitudes of the projections along the molecular axis and perpendicularly to it, respectively.

\begin{tabular}{|c|c|c|c|c|c|c|c|c|c|c|c|c|c|}
\hline \multirow{2}{*}{\multicolumn{2}{|c|}{ Structure Model }} & \multicolumn{4}{|c|}{ Static } & \multicolumn{4}{|c|}{ In-phase vibron } & \multicolumn{4}{|c|}{ Out-of-phase vibron } \\
\hline & & $q_{y}^{\mathrm{s}}(1)$ & $q_{z}^{\mathrm{s}}(1)$ & $q_{\|}^{\mathrm{s}}$ & $q_{\perp}^{\mathrm{s}}$ & $q_{y}^{\mathrm{i}}(1)$ & $q_{z}^{\mathrm{i}}(1)$ & $q_{\|}^{\mathrm{i}}$ & $q_{\perp}^{\mathrm{i}}$ & $q_{y}^{\mathrm{o}}(1)$ & $q_{z}^{\mathrm{o}}(1)$ & $q_{\|}^{\mathrm{o}}$ & $q_{\perp}^{\mathrm{o}}$ \\
\hline$C m c 2_{1}$ & $\mathrm{WF}$ & -0.044 & -0.003 & 0.037 & 0.023 & -0.172 & -0.037 & 0.161 & 0.071 & -0.138 & -0.030 & 0.129 & $\overline{0.057}$ \\
\hline (on-site) & EQ & -0.014 & -0.002 & 0.012 & 0.006 & -0.041 & -0.009 & 0.038 & 0.017 & 0.004 & -0.002 & 0.002 & 0.004 \\
\hline (off-site) & EQ & -0.017 & -0.007 & 0.018 & 0.003 & -0.050 & -0.023 & 0.054 & 0.009 & 0.004 & 0.000 & 0.003 & 0.002 \\
\hline$C 2 / m$ & $\mathrm{WF}$ & -0.073 & -0.013 & 0.073 & 0.013 & -0.338 & -0.078 & 0.344 & 0.045 & -0.437 & -0.086 & 0.439 & $\overline{0.073}$ \\
\hline (off-site) & EQ & -0.023 & 0.002 & 0.021 & 0.009 & -0.068 & 0.001 & 0.064 & 0.023 & 0.005 & -0.004 & 0.003 & 0.006 \\
\hline
\end{tabular}

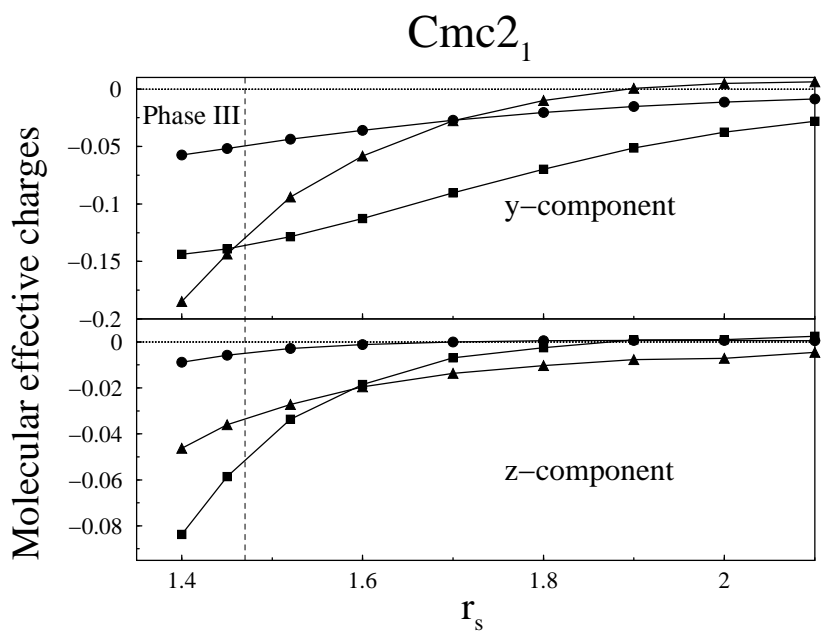

FIG. 3. Molecular effective charge vectors for molecule 1 in the hcp-centered (on-site) $C m c 2_{1}$ structure at several densities. - Static charge (Eq. 7); dynamical component of effective charge (last term in Eq. 9 ) for the in-phase vibron; $\boldsymbol{\Delta}$ dynamical component of effective charge for the out-of-phase vibron. The $x$-components vanish by symmetry.

produce the change in sign of $q_{z}^{\mathrm{s}}(n)$ for $C m c 2_{1}$ at low pressures 25]; other discrepancies can be seen in Table III, most notably in the vibron effective charges, and are discussed in Sec. IVB.

From Fig. 2 we can already see that $\left|\mathbf{q}^{\mathbf{s}}(n)\right|$ will be small, since the Wannier distribution is fairly symmetric with respect to the center of the paired protons. The dependence of the static charges versus $r_{s}$ is plotted in Figs. 3 and 1; as expected they vanish in the low density (large $r_{s}$ ) limit, and even at the highest pressures $(\sim 210 \mathrm{GPa})$ they are only a few percent of the electron charge, indicating that, at least in the structures under consideration, the ionicity of the molecules remains quite small, contrary to some proposals 27.28]. Notice also that at high pressures $\mathbf{q}^{\mathrm{s}}(n)$ becomes quite sensitive to the crystal structure (see Table IIII). This is not surpris-

\section{$\mathrm{C} 2 / \mathrm{m}$}

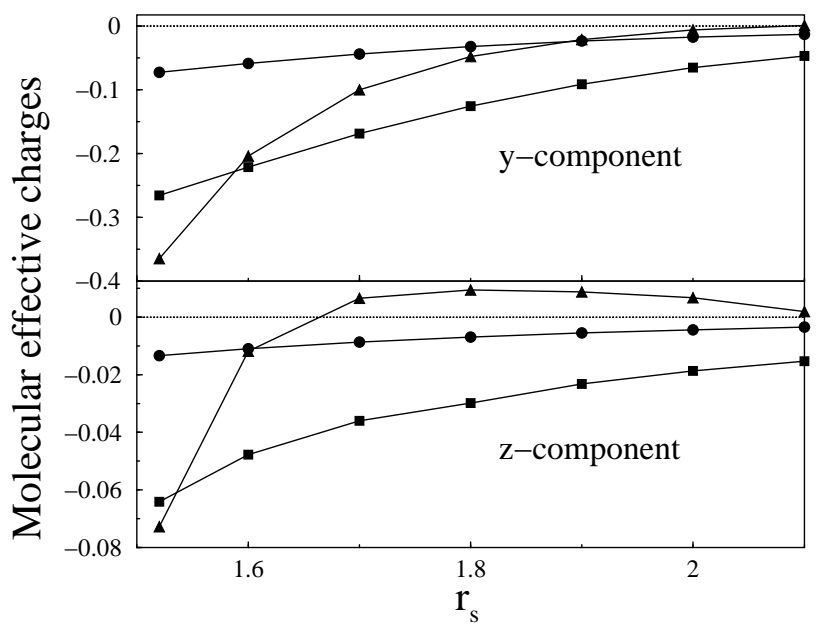

FIG. 4. Same as Fig. 3, but for the hcp-centered (on-site) $C 2 / m$ structure. We have only considered densities for which the LDA band gap remains open, which are below the density at which phase III appears.

ing, since it is totally induced by the crystal field. At $\sim 165 \mathrm{GPa}\left(r_{s}=1.45\right)$ the permanent dipole moment of an $\mathrm{H}_{2}$ Wannier molecule in the hcp-centered $C m c 2_{1}$ structure becomes 0.075 a.u., i.e. more than $1 / 10$ of the dipole of an isolated water molecule (0.74 a.u.).

\section{VIBRON INFRARED ACTIVITY}

\section{A. Wannier-function description}

The investigation of the number of IR-active lattice modes and their oscillator strengths in candidate structures is a useful guide in the search for the structures of the high-pressure phases 10,15,25]. Here we will focus on the vibron IR absorption observed in phase III 16,17]. Its 
relatively strong intensity is somewhat puzzling, since the stretching mode of the isolated $\mathrm{H}_{2}$ molecule is Ramanactive but IR-forbidden, and this has stimulated a large number of studies [10, 11, 17, 25, 28 30.

IR absorption is caused by the coupling of light to the change in $\mathbf{P}_{\text {mac }}$ induced by the lattice modes. In the basic theory of IR absorption in molecular crystals 19,31, the Clausius-Mossotti approximation of nonoverlapping molecules is assumed. The modern theory of polarization 23. treats rigorously the situation where that approximation breaks down, as discussed in the previous section. There, we decomposed the spontaneous macroscopic polarization into contributions from individual Wannier molecules; here we will do the same for the vibron-induced fluctuations in $\mathbf{P}_{\mathrm{mac}}$. For every vibron mode $\nu$ we will assign to each Wannier molecule a vibroninduced "effective charge" vector (compare with Eq. 7):

$$
\mathbf{q}^{\nu}(n)=\frac{\partial \mathbf{d}(n)}{\partial u_{\nu}}
$$

where $u_{\nu}$ is the normal coordinate [32]. The above expression is very similar to the definition of the Born effective charge associated with the stretching mode of a diatomic molecule [33]. The vector $\mathbf{q}^{\nu}(n)$ measures the vibron-induced symmetry-breaking charge transfer; with the help of Eq. 7, it can be decomposed into two parts (see Eq. 20 of Ref. 33] 34]:

$$
\mathbf{q}^{\nu}(n)=\mathbf{q}^{\mathrm{s}}(n)+u_{\nu} \frac{\partial \mathbf{q}^{\mathrm{s}}(n)}{\partial u_{\nu}} .
$$

Since $\mathbf{q}^{\mathrm{S}}(n)$ is the static charge, we will call the second term on the r.h.s. the dynamical charge.

Experimentally two vibrons have been detected in phase III: the lower frequency mode appears in the Raman spectrum, and the higher-frequency one in the IR spectrum [5]. Both the $C 2 / m$ and the $C m c 2_{1}$ structures have two vibron modes: one in which the two molecules in the primitive cell vibrate in-phase $(\nu=\mathrm{i})$, and a higher frequency mode in which they vibrate outof-phase $(\nu=0)$. The effective charges $\mathbf{q}^{\nu}(n)$ were calculated using Eq. 8 by changing the molecular bond length by small amounts $\delta u_{\nu}$ in the range $[0.0015,0.0035]$ a.u., after checking that such displacements yield essentially linear changes in the Wannier dipoles. Table III shows their values for $r_{s}=1.52$; as in the case of the static charges, we have in general that $q_{\|}^{\nu}>q_{\perp}^{\nu}$. Figs. 3 and 1 plot the static and dynamical charges versus $r_{s}$. Since these originate from the interactions between molecules, their magnitudes vanish in the low-pressure (large $r_{s}$ ) limit, and increase as pressure goes up. The most striking feature is that the dynamical terms increase with pressure much more rapidly than the static ones; at the highest pressures studied they are already 3.3 to 6.7 times larger, depending on the structure and on the vibron mode. Since this appears to be a rather general feature, it is also likely to occur in the yet-undetermined structure of phase III; the observed strong vibron IR activity is probably caused by this increase of the dynamical charges. Their dominant role had been previously inferred from the strong anisotropy of the atomic Born effective charge tensors 25.

Figs. 3 and 4 and Table III also show that the vibroninduced fluctuations in the individual molecular dipoles $\left(\mathbf{q}^{\nu}(n)\right)$, although clearly mode-dependent, are comparable for the two vibrons. (Interestingly, this is not so for the EQ model-see Section IV B.) The important difference occurs only after adding the contributions from the two molecules, and can be seen in Tables II and II. In the in-phase mode the large $y$-components cancel between the two molecules in the primitive cell, resulting in a weak IR activity (which actually vanishes in $\mathrm{C} 2 / \mathrm{m}$, since the small $z$-components also cancel). By contrast, in the out-of-phase mode the large $y$-components add up, resulting in a large net $\partial \mathbf{P}_{\mathrm{mac}} / \partial u_{\nu}$, and thus in a strong IR activity.

We emphasize again that all the quantities in Eq. 9 are gauge-dependent, like the Wannier functions themselves (but see Sec. VI). The gauge-invariant, measurable quantity is the net "vibron effective charge" vector, obtained by averaging $\mathbf{q}^{\nu}(n)$ over all molecules in a primitive cell:

$$
\overline{\mathbf{q}}^{\nu}=\frac{1}{M} \sum_{n=1}^{M} \mathbf{q}^{\nu}(n)=\frac{1}{n_{\mathrm{mol}}} \frac{\partial \mathbf{P}_{\mathrm{mac}}}{\partial u_{\nu}},
$$

where $n_{\text {mol }}$ is the number of molecules per unit volume; the vibron oscillator strength is proportional to $n_{\text {mol }}\left|\overline{\mathbf{q}}^{\nu}\right|^{2}$. The calculated values of $\left|\overline{\mathbf{q}}^{\nu}\right|$ versus $r_{s}$ are plotted in Fig. . together with the experimental results. For the out-of-phase mode in the $C m c 2_{1}$ structure the LDA calculation yields values very close to the experimentally measured IR absorption in phase III, but on the other hand the IR activity of the in-phase mode, although weaker, would still be observed, which is not the case. As for $C 2 / m$, the IR absorption is too strong compared to experiment. Hence it seems that neither structure is likely to be the correct one for phase III (this is also supported by the large number of observed libron modes in phase III [35, 36], which is incompatible with structures with such small primitive cells). Nevertheless, the above results for these structures allow us to make an important general point. They show that large permanent molecular dipoles are not required in order for strong vibron IR absorption to occur, contrary to what has been sometimes stated in the literature [11,28, 37. In fact, in both structures the magnitude of the permanent dipoles (static charge) is far too small to account by itself for the measured absorption. However, once the dynamical charge transfer is accounted for, the resulting IR activity becomes even larger than the one measured in phase III.

Finally, Table III and Fig. 5 show that the displacement away from the hcp sites significantly increases the vibron-induced charges, as well as the static charges. This is yet another example of the strong sensitivity of 


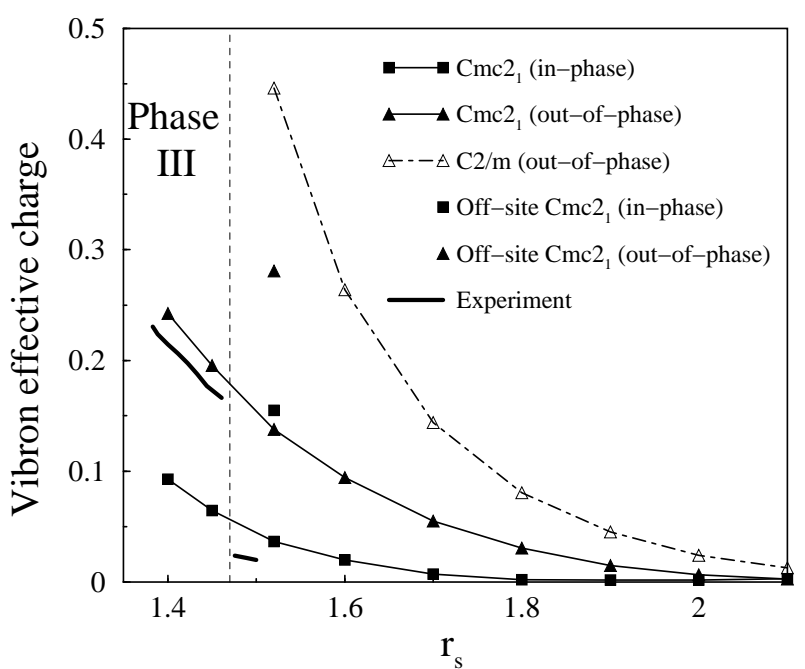

FIG. 5. Magnitude of the net vibron-induced effective charge vector $\left|\overline{\mathbf{q}}^{\nu}\right|$ (Eq. 10) versus $r_{s}$, for the in-phase and out-of-phase vibron modes in the $C m c 2_{1}$ and $C 2 / m$ structures. Experimental data for phases II and III is from Ref. [17, and was converted from Szigeti to Born charges [38].

the effective charges to the crystal structure, which may help explain the large difference in the intensity of IR absorption between phase III and the lower pressure phases [25, 10.

\section{B. Comparison with the EQ model}

Table III also contains the values of the vibron-induced charge vectors on each molecule, as calculated from the EQ model [26]. Like the static charges, they are significantly smaller than those obtained from the WFs. Another important difference is that in the EQ model the vibron-induced charges on the individual molecules are more than one order of magnitude smaller for the outof-phase than for the in-phase mode, whereas their WF counterparts are comparable for the two vibrons. The reason is the following: in the EQ model the vibroninduced change in a molecular dipole can be written to first order as

$$
\delta d_{\|} \simeq \delta \alpha_{\|} E_{\|}+\alpha_{\|} \delta E_{\|},
$$

where $\alpha$ is the molecular polarizability and $E$ is the quadrupolar electric field on the molecular site (for definiteness we look at the dipole along the molecular axis; the same analysis applies to the perpendicular component). The first term on the r.h.s. is equal for the two vibrons, so that the difference between their effective charges arises from the second term. Choosing the isolated-molecule parameters from Refs. [39] and 40], it turns out that the two terms have a very similar magnitude. But whereas in the in-phase mode they have the same sign, in the out-of-phase mode they have opposite

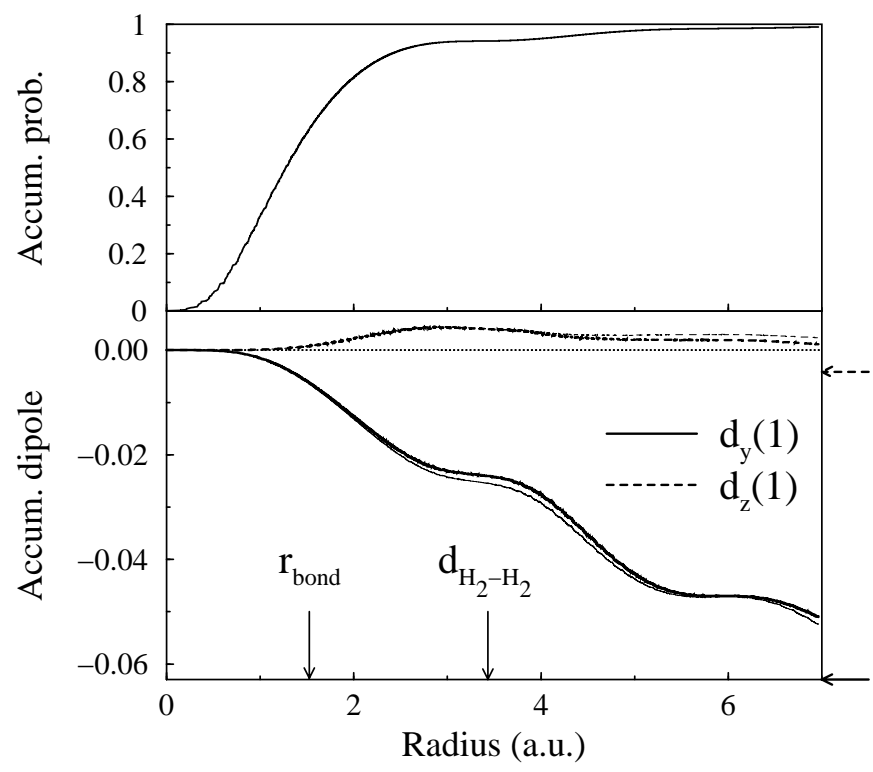

FIG. 6. Modulus squared (upper panel) and dipole (lower panel) of a WF, accumulated by integrating up to a certain radius around the molecular center, for $C m c 2_{1}$ at $r_{s}=1.52$. The $x$-component of the dipole vanishes by symmetry independent of radius, and the horizontal arrows denote the converged values. The thin lines correspond to the WF obtained from bond-centered Gaussians with a r.m.s. width of $2.0 \AA$ (see Sec. II).

signs, so that their contributions largely cancel, resulting in a much smaller molecular effective charge. As a consequence, in the $C m c 2_{1}$ structure the in-phase oscillator strength comes out larger than the out-of-phase, which is the opposite of the LDA result. These discrepancies between the LDA WFs and the EQ model are likely to be related at least in part to the rather delocalized nature of the induced dipoles, which will be discussed in the next section, whereas the EQ model assumes point-like (infinitely localized) molecules.

\section{SPATIAL EXTENT OF THE WANNIER MOLECULES}

In the previous sections we focused our attention on information that can be extracted from the location of the centers of charge of the WFs. Here we will examine in detail their spatial distribution at high pressure, in particular their spatial extent. This will allow us to investigate the effects associated with the overlap between neighboring WFs; such effects are expected to be significant at megabar pressures, as suggested by the large bandwidths, in excess of $20 \mathrm{eV}$ (see Fig. 12). 


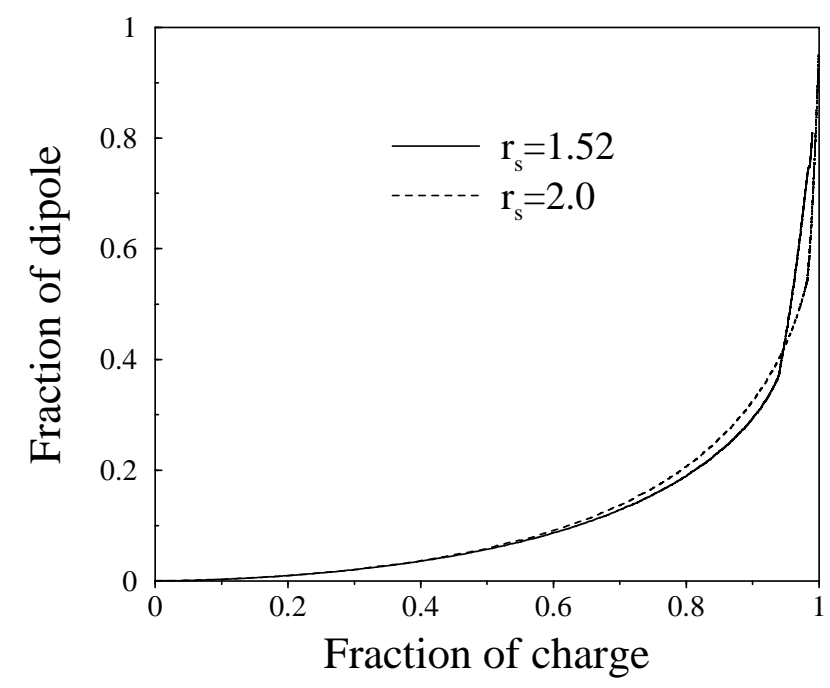

FIG. 7. Fraction of the Wannier dipole $d_{y}(1)$ accumulated by integrating up to a certain radius around the molecular center, versus the fraction of the Wannier charge that lies inside the same radius, for $C m c 2_{1}$ (see also Fig. 6).

\section{A. Spread of the Wannier charge and dipole distributions}

The spread of the Wannier charge and dipole distributions are presented in Fig. 6. For $C m c 2_{1}$ at $r_{s}=1.52$ $\left(r_{s}=2.0\right)$, a radius of 1.72 a.u. $(2.25$ a.u. $)$, half the shortest intermolecular distance $d_{\mathrm{H}_{2}-\mathrm{H}_{2}}$, encloses about $72 \%(85 \%)$ of the charge and only $17 \%(28 \%)$ of the $y$ component of the dipole. This suggests that already at $r_{s}=2.0(\sim 13 \mathrm{GPa})$ the overlap between nearby WFs is far from negligible. It is also clear that the dipole is significantly more spread out than the charge, with very large contributions arising from the orthogonality tails in the overlap region, where the Wannier charge density is very small. The longer range of the dipole is to be expected, due to the factor $\mathbf{r}$ in Eq. 6, but the large magnitude of the effect is somewhat surprising. Fig. I shows even more clearly that for both pressures a rather small fraction of the total charge, located in the overlapping tails of the $\mathrm{WF}$, is responsible for most of the dipole. Also striking is the fact that, at $r_{s}=1.52$, up to a radius of 7 a.u. the accumulated $d_{z}(1)$ remains positive, whereas the converged value is negative; this suggests that the agreement in sign with the EQ model (see Table III) may be fortuitous, since in that model the dipole is caused by the electric field at the center of the molecule.

By analogy with the radially integrated Wannier charge and dipole distributions (Fig. (6), one can plot the derivative of these quantities with respect to the normal coordinate of a vibron mode (Fig. 8). At high pressures the contributions from the overlapping tails to the change in the dipole moment are very significant, even more so than for the equilibrium dipole. In other words, in the

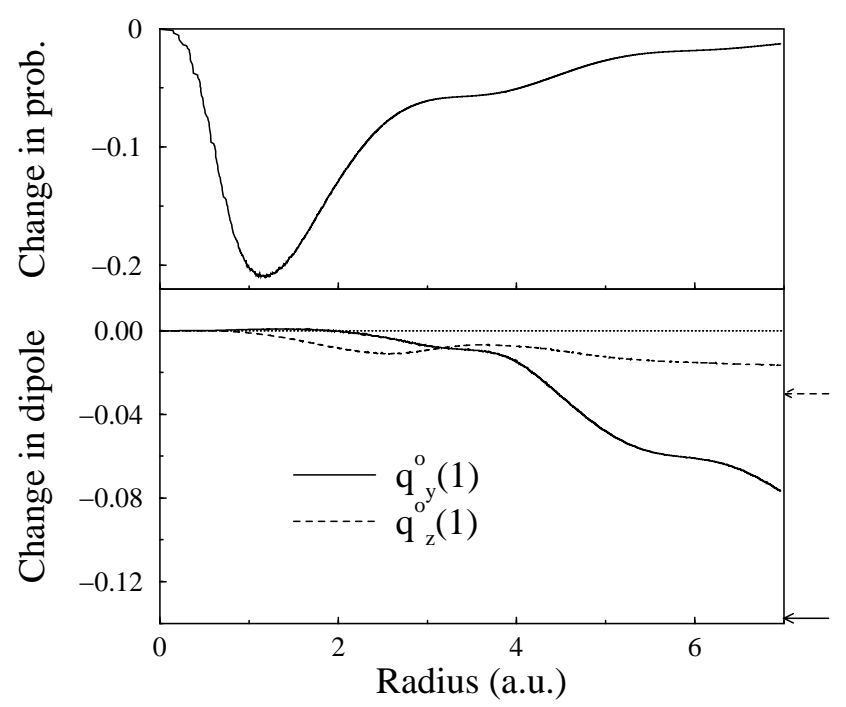

FIG. 8. Upper panel: derivative with respect to the out-of-phase vibron's normal coordinate $u_{\mathrm{o}}$ of the accumulated probability plotted in the upper panel of Fig. 6. Lower panel: derivative with respect to $u_{\mathrm{o}}$ of the accumulated dipole plotted in the lower panel of Fig. 6, i.e., accumulated radial integral of the vibron-induced effective charge vector $\mathbf{q}^{\circ}(1)$ (Eq. 8) (the $x$-component vanishes by symmetry for all radius). The arrows denote the converged values.

dense solid the dynamical charge transfer processes responsible for the IR activity are very delocalized.

The effect of a vibron on the Wannier charge distribution is depicted in the upper panel of Fig. 9: charge is depleted from the inner part and accumulates in the outer part of the molecule. Note that the charge transfer occurs mainly along the molecular axis, and is essentially symmetrical with respect to the molecular center, as one would expect from stretching an isolated molecule, which does not break the symmetry between the two atoms. This kind of charge transfer alone would lead to a zero net change in the (vanishing) molecular dipole, and hence to no IR absorption. The contribution of a Wannier molecule to the IR activity of the crystal comes from the comparatively small odd part (with respect to its center) of the charge transfer. Since this is barely visible in the upper panel of Fig. 9, in the lower panel we have removed the large even part. It is interesting to note that near the paired protons most of the odd part is oriented roughly perpendicularly to the molecular axis, making a small angle with the $c$ axis, such that it gives a small positive contribution to $q_{y}^{\mathrm{o}}(1)$ and a larger negative contribution to $q_{z}^{\circ}(1)$. This observation is supported by Fig. 8, which shows that for small radius the accumulated $q_{z}^{\mathrm{o}}(1)$ is negative and larger than the accumulated $q_{y}^{\mathrm{o}}(1)$, which is positive. For large radius $q_{y}^{\mathrm{o}}(1)$ changes sign and ends up overtaking $q_{z}^{\mathrm{o}}(1)$, and the net molecular vibron charge vector has a larger projection along the molecular axis than perpendicularly to it (see Table III). 

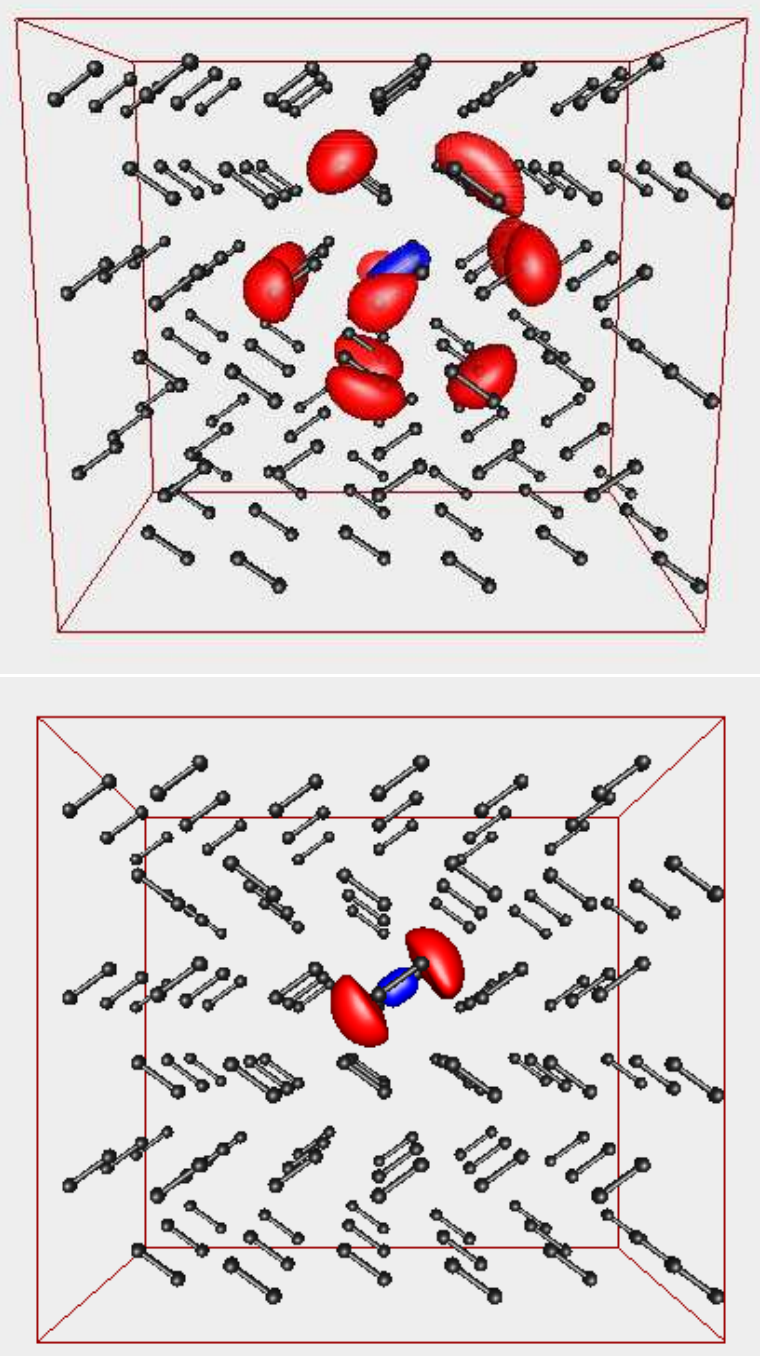

FIG. 9. Upper panel: derivative of the modulus squared of the Wannier orbital with respect to the normal coordinate of the out-of-phase vibron mode $\left(v \partial\left|w_{1}(\mathbf{r})\right|^{2} / \partial u_{\mathrm{o}}\right)$ for $C m c 2_{1}$ at $r_{s}=1.52(v=59.3 \mathrm{a} . \mathrm{u}$. is the volume of the primitive cell). The central contour has an amplitude of -4.5 , and the two outer contours have an amplitude of +0.25 . Lower panel: odd part (with respect to the center of the molecule) of the same quantity. The upper (lower) contour has an amplitude of $+0.05(-0.05)$.
The results of this section should be relevant for models that attempt to account for the dielectric properties of compressed hydrogen. For instance, it seems unlikely that models based on point-like objects, such as the electric quadrupole (EQ) model [10,17,29], contain all the important ingredients that lead to the strong IR absorption in the highly compressed phase III. The reasons are twofold: at such high densities (i) the electrostatic interactions are expected to differ substantially from the ideal quadrupolar one 41] (and in fact the validity of a multipole expansion becomes questionable when the molecular charges overlap significantly), and (ii) the "classical" treatment of polarization, based on the bulk $\rho(\mathbf{r})$, becomes inadequate [23]. We note that although the EQ model can account for both static and dynamical charges [17,29, 31 (see Sec. IV B), both effects are then due to local fields and polarizabilities (see also Fig. 2 of Ref. 33 and associated discussion regarding local versus nonlocal mechanisms).

The central conclusion of the preceding analysis is that the contributions to the induced molecular dipoles (and their fluctuations) arising from the overlapping tails of the Wannier orbitals, which extend well beyond the nearest neighbor molecules, are crucial. It is instructive to contrast this state of affairs with what happens in liquid water: there, the contribution from the orthogonality tails is negligible [42]. This difference may stem from the fact that an isolated water molecule is polar, so that the effect of the liquid environment is only to modify a previously existent dipole moment, whereas in solid hydrogen the molecular dipole is totally induced. Induced dipoles tend to be rather extended because the outer regions of the molecules are most easily polarizable [43; thus their contribution to the dipole can be large, even though $\rho(\mathbf{r})$ is small, because of the $\mathbf{r}$ factor in Eq. 6 . In conclusion, the WF analysis strongly suggests that the Clausius-Mossotti picture of nonoverlapping dipoles breaks down rather dramatically for solid hydrogen at megabar pressures.

\section{B. Measuring the molecular overlap}

The overlap between the charge distributions of neighboring WFs can be quantified as 44,42

$$
O_{m n}=\frac{\int\left|w_{m}(\mathbf{r})\right|^{2}\left|w_{n}(\mathbf{r})\right|^{2} d \mathbf{r}}{\left(\int\left|w_{m}(\mathbf{r})\right|^{4} d \mathbf{r}\right)^{1 / 2}\left(\int\left|w_{n}(\mathbf{r})\right|^{4} d \mathbf{r}\right)^{1 / 2}} .
$$

For $C m c 2_{1}$ the largest value of $O_{m n}$ is 0.005 at $r_{s}=2.0$ and 0.021 at $r_{s}=1.52$; the latter value is still quite small, roughly twice the value for WFs located on nearby water molecules in liquid water 42,44. Thus, by inspection of $O_{m n}$ alone one would not suspect that the overlapping tails are so much more important for the dipole moments in compressed solid hydrogen than in liquid water. The reason is that $O_{m n}$ measures the overlap between charge 


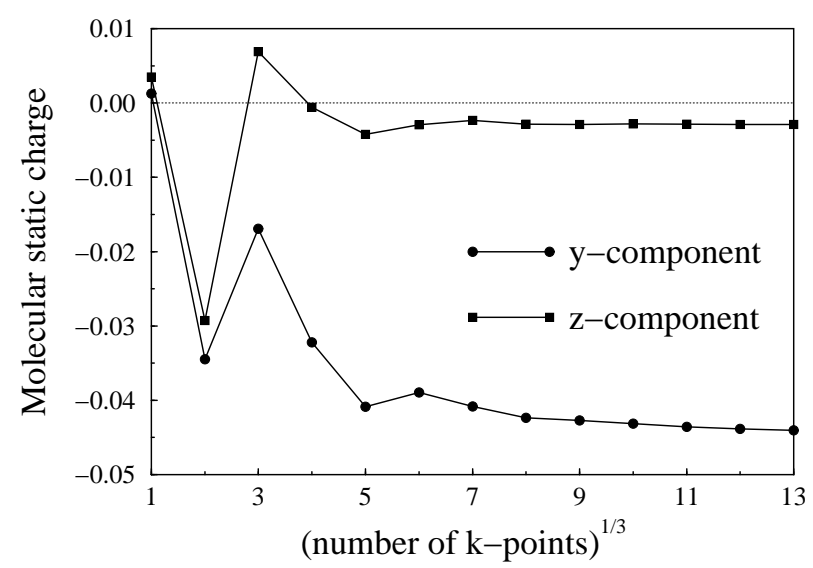

FIG. 10. Static charge vector $\mathbf{q}^{\mathrm{s}}(1)$ (Eq. 7) versus $n$, where $n \times n \times n$ is the size of the mesh of $k$-points used for computing the WFs, for the on-site $C m c 2_{1}$ at $r_{s}=1.52$. The number of $k$-points is kept fixed at $11 \times 11 \times 11$ during the self-consistent calculation.

distributions, whereas in this system the dipoles are much more spread out.

Another indication that overlap effects are important comes from the well-known fact that a very large number of $k$-points is required to converge the total-energy calculations in compressed hydrogen [41]. In fact, if the molecules were strictly nonoverlapping a single $k$-point would suffice for computing all physical properties. In Fig. 10 is shown the static charge (i.e., the dipole) for WFs obtained using different meshes of $k$-points. It is clear that a dense mesh is required for converging this quantity. This results from the fact that, when using a discrete mesh, the WFs are actually periodic in real space, with a periodicity which is inversely proportional to the spacing between neighboring points [3]. Hence the need for a fine sampling of the Brillouin zone is just a manifestation of the large contributions to the Wannier dipole arising from the tails far away from the "home" unit cell. In solid hydrogen a dense mesh of $k$-points is expected to be even more important for the dielectric properties than for the total energy, since $\mathbf{P}_{\text {mac }}$ is particularly sensitive to the Wannier tails.

\section{Quadratic spread and localization length}

Another way of quantifying the spatial extent of the WFs is in terms of their quadratic spread. According to Eq. A, the r.m.s. width of the Wannier probability distribution, averaged over all three Cartesian directions and over the occupied WFs, is $\bar{\lambda}=(\Omega / 3 M)^{1 / 2}$. This quantity is plotted versus $r_{s}$ in Fig. 11. Notice that it increases with increasing pressure (decreasing $r_{s}$ ), i.e., the Wannier molecules become more extended upon compression, which is the opposite of what happens in the usual mod-

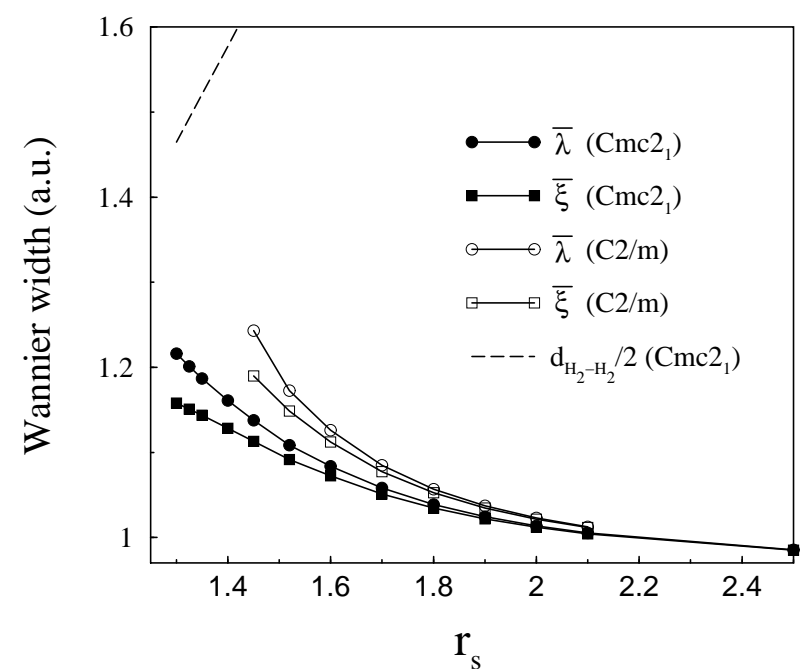

FIG. 11. Root-mean-square width $\bar{\lambda}=(\Omega / 3 M)^{1 / 2}$ of the WFs and electronic localization length $\bar{\xi}$, both averaged over all directions. The dashed line denotes half the shortest intermolecular distance in $C m c 2_{1}$ (in $C 2 / m$ it is very similar).

els of molecular solids [45,46]. This is an overlap effect, caused by the orthogonality requirement. It is due to the enhancement of the outer corona shown in Fig. 2, which is not included in the definition of "molecules" in those models. It can also be viewed as a result of the gap reduction with pressure.

Also plotted in Fig. 11 is the electronic localization length $\bar{\xi}=\left(\Omega_{\mathrm{I}} / 3 M\right)^{1 / 2}[47,48]$, where $\Omega_{\mathrm{I}}<\Omega$ is the gauge-invariant part of the spread of the WFs [3]. $\bar{\xi}^{2}$ measures the mean-square quantum fluctuation of the macroscopic polarization [48], normalized in such a way as to be finite for insulators, and diverging when the band gap closes. Notice that in the low-density limit $\bar{\lambda} \rightarrow \bar{\xi}$; that happens because there is only one occupied WF per molecule, and can be understood by comparing Eqs. 14 and 15 of Ref. [3]. As expected, at high pressures $\bar{\lambda}$ and $\bar{\xi}$ are larger for the $C 2 / m$ structure, which has the smaller band gap: at $r_{s}=1.52 \bar{\lambda}$ has increased by $13 \%(20 \%)$ in $C m c 2_{1}(C 2 / m)$ with respect to the low-density (isolated molecule) value.

\section{UNIQUENESS OF WELL-LOCALIZED WANNIER MOLECULES}

As discussed earlier, the WFs are strongly nonunique; in particular, it is only the sum of all the Wannier dipoles over a primitive cell which is physically meaningful (Eq. 5), whereas the individual dipoles (and hence $\mathbf{q}^{\mathbf{S}}(n)$ and $\left.\mathbf{q}^{\nu}(n)\right)$ are gauge-dependent. Nevertheless, in this work we have been looking at the individual dipoles of the "maxloc" WFs in an attempt to extract from them useful physical information. The underlying assumption 
is that in practice well-localized WFs are fairly unique. For solid hydrogen this is obviously true in the low density limit, where they reduce to the bonding orbitals of the individual molecules. Here we will discuss to what extent that assumption holds for the compressed solid as well.

A systematic way of assessing the degree of uniqueness of well-localized WFs would be to implement different localization criteria and then compare the resulting "maximally-localized" WFs. We have not attempted such a detailed study; instead we have performed a simpler test, which is a first step in that direction. As mentioned in Sec. II, in the method we are using [3] an initial guess is made for the localized WFs, with the help of "trial functions", which in our case are bond-centered Gaussians (we will call the resulting orbitals "projected WFs"). Their localization is then enhanced by minimizing the quadratic spread $\Omega$ (Eq. 乘), yielding the "maxloc" WFs. Since the projected WFs are totally oblivious of the localization criterion that one later uses to further localize them, it seems reasonable to assume that the difference between the projected and the "maxloc" WFs is an upper bound to the differences that would occur between WFs obtained using any two "sensible" localization criteria.

Let us consider the $C m c 2_{1}$ structure at $r_{s}=1.52$, for which the average r.m.s. width of the "maxloc" WFs is $\bar{\lambda}=1.11$ a.u. If we choose the r.m.s. width of the initial bond-centered Gaussians to be 1.89 a.u. (1 $\AA$ ), the resulting projected WFs are essentially indistinguishable from the "maxloc" ones: for instance, the curves corresponding to those in Fig. 6 are virtually identical, and the individual Wannier dipoles remain the same to at least six significant digits! This is compelling evidence for a high degree of uniqueness of well-localized WFs in this system, at least for the high-symmetry configurations that we studied. If we double the width of the initial Gaussian, some differences start to appear. They remain barely visible in the accumulated radial integral of the probability (upper panel of Fig. 6) but are noticeable, although still relatively small, in the radially integrated dipole (lower panel of Fig. 6). For instance, the large $y$-component of the dipole changes by around $2 \%$.

\section{TIGHT-BINDING ANALYSIS}

In this section, we investigate whether the essential physics of the WFs in high-pressure $\mathrm{H}_{2}$ phases can be captured by a simpler tight-binding (TB) approach. As is well known, the TB approximation provides a simple, computationally inexpensive method for computing electronic structure effects, and has the additional advantage that its output is easily interpreted in terms of a local, real-space picture [49]. Thus, TB is a natural approach to explore here, where we want to study the dielectric properties of $\mathrm{H}_{2}$ phases from just this kind of local point of view. We confirm below that a previously-proposed $s p^{3}$ TB model [50] provides a good description of the occupied bands in these systems, and show how the operations of constructing the WFs and computing their contributions to dielectric properties (such as electric polarization) can be carried out in the TB framework. Finally, using the fact that the TB representation automatically provides an atom-by-atom and orbital-by-orbital decomposition, we obtain useful insights into the nature of the WFs and their contributions to the dielectric properties.

\section{A. Tight-binding formalism}

In the TB method, the Bloch functions $\psi_{n \mathbf{k}}$ are expanded in a basis of atomic-like orbitals $\phi_{i l}$ as

$$
\psi_{n \mathbf{k}}(\mathbf{r})=\sum_{i l} C_{n \mathbf{k}}(i l) e^{i \mathbf{k} \cdot \mathbf{r}_{i l}} \phi_{i l}(\mathbf{r}) .
$$

Here $l$ labels the unit cell located at $\mathbf{R}_{l}, i$ labels an orbital on the atom at $\mathbf{r}_{i l}=\mathbf{R}_{l}+\tau_{i}$ (where $\tau_{i}$ specifies the relative position of the atom within the unit cell), and the vector of coefficients $C_{n \mathbf{k}}(i l)$ forms the TB representation of the Bloch function. Our goal is to carry out a unitary transformation to a set of $M$ localized WFs

$$
w_{\alpha}(\mathbf{r})=\frac{1}{N} \sum_{n \mathbf{k}} U_{\alpha n}^{(\mathbf{k})} \psi_{n \mathbf{k}}(\mathbf{r})
$$

associated with a set of $M$ occupied bands, where the $U_{\alpha n}^{(\mathbf{k})}$ are $\mathbf{k}$-dependent $M \times M$ unitary matrices that will be fixed by the requirement of maximal localization [3]. Introducing the TB representation of the WF

$$
w_{\alpha}(\mathbf{r})=\sum_{i l} W_{\alpha}(i l) \phi_{i l}(\mathbf{r})
$$

it follows that

$$
W_{\alpha}(i l)=\frac{1}{N} \sum_{n \mathbf{k}} U_{\alpha n}^{(\mathbf{k})} e^{i \mathbf{k} \cdot \mathbf{r}_{i l}} C_{n \mathbf{k}}(i l) .
$$

The essential ingredients needed for the construction of the maximally-localized WFs [3], or for the computation of the Berry-phase polarization [23], are inner products $\left\langle u_{n \mathbf{k}} \mid u_{n^{\prime} \mathbf{k}^{\prime}}\right\rangle$ between the cell-periodic part of the Bloch functions

$$
u_{n \mathbf{k}}(\mathbf{r})=e^{-i \mathbf{k} \cdot \mathbf{r}} \psi_{n \mathbf{k}}(\mathbf{r})
$$

at nearby $k$-points in the Brillouin zone. In principle, the calculation of the $\left\langle u_{n \mathbf{k}} \mid u_{n^{\prime} \mathbf{k}^{\prime}}\right\rangle$ requires a detailed knowledge of the basis orbitals $\phi_{i l}$, which has been done in Ref. [51]. However, in the spirit of minimal empirical TB, we make the approximation

$$
\left\langle u_{n \mathbf{k}} \mid u_{n^{\prime} \mathbf{k}^{\prime}}\right\rangle=\sum_{i l} C_{n \mathbf{k}}^{*}(i l) C_{n^{\prime} \mathbf{k}^{\prime}}(i l) .
$$


[When completing the circuit across a Brillouin zone boundary, the relation $C_{n, \mathbf{k}+\mathbf{G}}(i l)=e^{-i \mathbf{G} \cdot \mathbf{r}_{i l}} C_{n \mathbf{k}}(i l)$ should be used to translate by a reciprocal lattice vector $\mathbf{G}$.]

Eq. (18) can be derived via a Taylor expansion of the exponential factor $\exp \left[i \mathbf{k} \cdot\left(\mathbf{r}-\mathbf{r}_{i l}\right)\right]$, with the following assumptions: (i) that the TB basis orbitals are orthonormal, $\left\langle\phi_{i l} \mid \phi_{i^{\prime} l^{\prime}}\right\rangle=\delta_{i i^{\prime}} \delta_{l l^{\prime}}$; (ii) that the position operator is diagonal in the TB basis, $\left\langle\phi_{i l}|\mathbf{r}| \phi_{i^{\prime} l^{\prime}}\right\rangle=\mathbf{r}_{i l} \delta_{i i^{\prime}} \delta_{l l^{\prime}}$; and (iii) that matrix elements of higher powers of the position operator are likewise trivial,

$$
\left\langle\phi_{i l}\left|x^{p} y^{q} z^{r}\right| \phi_{i^{\prime} l^{\prime}}\right\rangle=x_{i l}^{p} y_{i l}^{q} z_{i l}^{r} \delta_{i i^{\prime}} \delta_{l l^{\prime}} .
$$

Conditions (i) and (ii) are actually special cases of (iii) and all all are quite artificial in that they cannot be satisfied for actual basis functions. For example, while an $s p^{3}$ hybrid on a given atom should have its charge center displaced from the geometric center of the atom, condition (ii) does not allow this effect to be captured. Similarly, the spread $\left\langle\phi_{i l}\left|r^{2}\right| \phi_{i l}\right\rangle-\left\langle\phi_{i l}|\mathbf{r}| \phi_{i l}\right\rangle^{2}$ of an individual basis orbital is taken to vanish, according to condition (iii). Nevertheless, Eq. (18) is the logical extension of the empirical TB philosophy, in which one tries to avoid introducing any additional parameters beyond those needed to parameterize the Hamiltonian itself. Despite its simplicity, the tests presented below demonstrate that this approach captures much of the interesting complexity of the WFs, at least for the systems under study here. A similar approximation was previously shown to allow for reasonably accurate TB calculations of dynamical effective charges in semiconductors [52].

In practice, we work entirely within the TB representation. First the $C_{n \mathbf{k}}(i l)$ are determined on a regular mesh of $k$-points by solving the standard secular equation involving the Hamiltonian matrix $H_{i i^{\prime}}^{(\mathbf{k})}$. Then the electric polarization can be computed by inserting Eq. (18) into the formalism of Ref. 23]. Similarly, an "optimal" set of unitary matrices $U_{\alpha n}^{(\mathbf{k})}$ can be obtained by inserting Eq. (18) into the formalism of Ref. [3], and from these, the WFs $W_{\alpha}(i l)$ obtained via Eq. (16). The resulting WFs are optimal in the sense of being maximally localized in real space, i.e., of minimizing Eq. (1).

In the "maxloc" method [3], one usually begins by choosing a set of localized "trial functions," and making a preliminary unitary rotation among the Bloch orbitals in order to maximize their projections onto these trial functions, as discussed in the LDA context at the end of Sec. II. In the TB context, we have found the following natural way of constructing the trial Wannier functions. Since we have two molecules per cell, we want to carry out the $2 \times 2$ rotation that makes one state have most of its projection on the first molecule, and the other have most of its projection on the second molecule. To do this, we consider the difference $\Delta \mathbf{P}=\mathbf{P}_{1}-\mathbf{P}_{2}$ of projection operators $\mathbf{P}_{1}$ and $\mathbf{P}_{2}$ onto the first and second molecule, respectively. (In the TB basis, $\Delta \mathbf{P}$ is just a diagonal matrix with \pm 1 diagonal entries.) Then, at
TABLE IV. Tight-binding parameters of Ref. [50], in eV.

\begin{tabular}{ccc}
\hline \hline Hopping parameters & Intramolecular & Intermolecular \\
\hline$V_{s s}$ & -8.50 & -0.04 \\
$V_{s p}$ & -8.75 & -0.16 \\
$V_{p p \sigma}$ & +9.00 & +0.89 \\
\hline \hline
\end{tabular}

each $\mathbf{k}$, we diagonalize $\Delta \mathbf{P}$ in the space of the two Bloch states, and set the phase of each eigenvector by requiring that its inner product with an even linear combination of $s$ orbitals on the two atoms comprising the molecule should be real and positive. We find that the unitary transformation $\widetilde{U}_{\alpha n}^{(\mathbf{k})}$ obtained in this way turns out to be an excellent approximation to the "maxloc" $U_{\alpha n}^{(\mathbf{k})}$ which minimizes Eq. (1). In fact, subsequent minimization typically only leads to changes of WF coefficients of order one part in $10^{-5}$, and so that in practice it is not even necessary to carry out the maxloc minimization procedure. This is consistent with our similar experience in the LDA context as discussed at the end of Sec. V1.

\section{B. Details of the tight-binding model}

The TB parameterization we used is the one proposed by Chacham et al. [50]. These authors showed that the main characteristics of the electronic structure of highdensity solid hydrogen at megabar pressures could be reproduced by using a minimal orthogonal TB basis comprised of $s, p_{x}, p_{y}$, and $p_{z}$ orbitals on each hydrogen atom. The intermolecular matrix elements are taken as

$$
\begin{aligned}
V_{s s} & =V_{s s}\left(d_{0}\right) e^{\alpha\left(1-d / d_{0}\right)} \\
V_{p p \sigma} & =V_{p p \sigma}\left(d_{0}\right) e^{\beta\left(1-d / d_{0}\right)} \\
V_{s p} & =V_{s p}\left(d_{0}\right) e^{[(\alpha+\beta) / 2]\left(1-d / d_{0}\right)} \\
V_{p p \pi} & =0
\end{aligned}
$$

with dimensionless constants $\alpha=5.76$ and $\beta=2.52$, where $d$ is the interatomic distance and $d_{0}=3.79 \AA$ is the equilibrium hcp lattice constant at zero pressure. Given in Table IV] are the $V_{s s}\left(d_{0}\right), V_{p p \sigma}\left(d_{0}\right), V_{s p}\left(d_{0}\right)$, and the intramolecular matrix elements $V_{s s}, V_{s p}$ and $V_{p p \sigma}$ which are independent of $d$. The intra-atomic parameter $\epsilon_{s}-$ $\epsilon_{p}=-20 \mathrm{eV}$.

In addition to this original tight-binding scheme, we have also tested an extended scheme that includes a correction designed to incorporate the effects of the quadrupolar electrostatic fields arising from neighboring molecules. In this extended "TB+Q" scheme, the molecules are first modeled as point quadrupoles centered at the molecular sites (mid-bond positions). The quadrupole moment tensor for each molecule is taken from the free-molecule calculations of Ref. [39] by assuming a linear dependence upon the bond length in the range of 1.4-1.6 a.u. The total quadrupolar electric field 
is then evaluated at each molecular site, and the electrostatic potential shift on each atom in the molecule is calculated by assuming a linear extrapolation to the atomic position. Finally, the diagonal elements (self-energies) of the TB Hamiltonian matrix are modified by adding these energy shifts, and the solution of the secular equation then proceeds as usual.

\section{Band structure}

We have applied the TB model to the same $C m c 2_{1}$ and $C 2 / m$ candidate $\mathrm{H}_{2}$ structures studied with LDA methods in earlier sections, and confirmed that this TB model does a good job of reproducing the critical features of the electronic band structure. For convenience, we present only results on the $C m c 2_{1}$ geometry; the corresponding results for $C 2 / \mathrm{m}$ are qualitatively similar. As already indicated in Sec. IC, our $C m c 2_{1}$ structure has $r_{s}=1.52$ a.u., $r_{\text {bond }}=1.445$ a.u, $c / a=1.576$, and the tilt angle $\theta=54.0^{\circ}$. The two-molecule (four-atom) unit cell is illustrated in Fig. 1. Use of the $s p^{3}$ TB basis leads to a $16 \times 16$ TB Hamiltonian matrix.

Figure 12 shows the good agreement between TB and LDA band structures for this geometry. The agreement in the occupied valence-band region (lowest two bands) is excellent, and the resemblance in the conduction-band region is also reasonable. The TB model predicts a gap closure at a density of $0.3962 \mathrm{~mol} / \mathrm{cm}^{3}$, consistent with the results from other studies [53]. The band structure is hardly affected at all if the $\mathrm{TB}+\mathrm{Q}$ theory is used in place of simple TB.

The WFs are constructed for the two occupied bands by using the definition in Eq. 16. A $10 \times 10 \times 10 k$-point mesh is used in calculation. Since there are only two WFs in the unit cell, and these are related to each other by a symmetry, it suffices to analyze just one of them. In Table $\mathrm{V}$ we analyze the spatial distribution of the WF by decomposing into contributions coming from the "home molecule" and the first two nearest-neighbor shells of molecules in real space. The home molecule is labeled as "Neighbor 0", the next six neighboring molecules form the first shell at a radius of 3.4342 a.u. (0.9767 in units of lattice constant), and the second shell comprises the next six neighboring molecules at a radius of 3.5162 a.u. (one lattice constant). Table $\mathrm{V}$ gives a clear picture of the spatial structure of the WFs. We can see that the WFs have about $85 \%$ of their probability on the home molecule, 91\% (cumulatively) inside the first shell, and $97 \%$ up to the second shell. In the TB framework, the contribution to the WFs can be very easily decomposed further into the $s p_{3}$ tight-binding basis orbitals, as shown in Fig. 13 . While the $s$-orbital contribution is $\sim 5$ times larger than that of the $p$ orbitals, we find that the $s$ contribution is almost entirely localized to the home molecule. On the other hand, although the $p$ orbitals give a smaller total contribution, they play a much bigger role in the tail re-
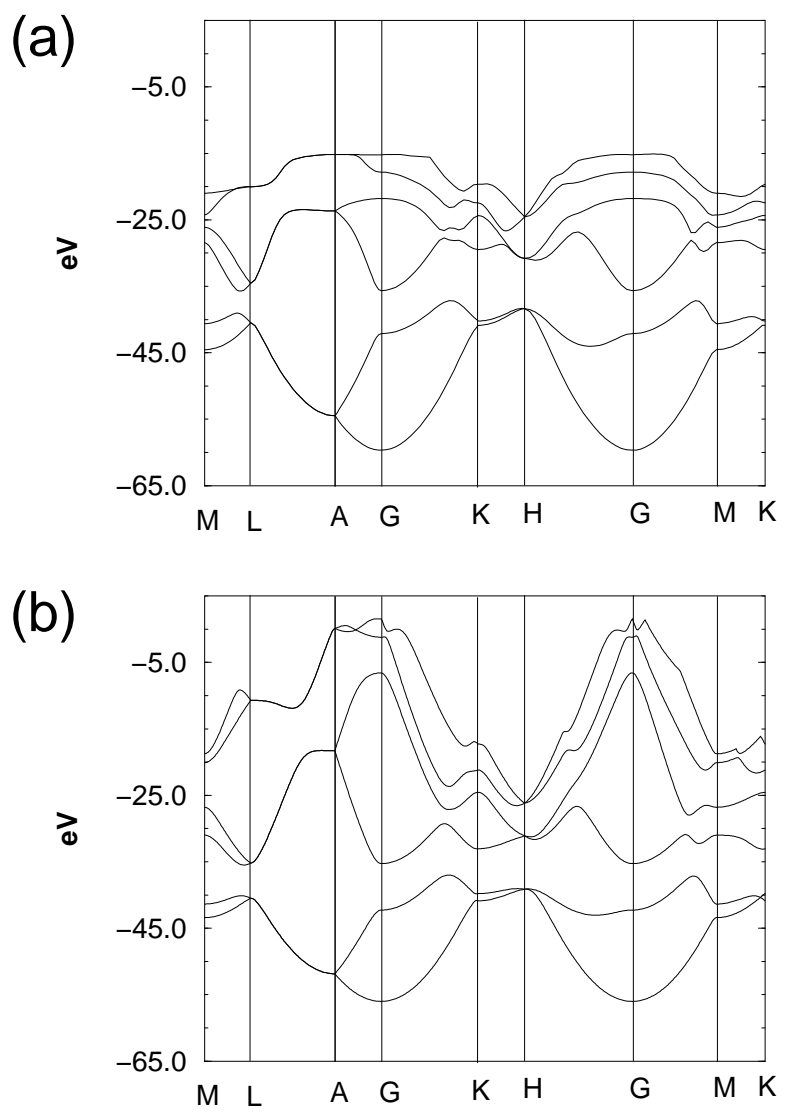

FIG. 12. Electronic band structure calculated by (a) tight-binding method, and (b) LDA approach.

gion that determines the spatial distribution of the WFs.

\section{Tight-binding Wannier functions}

The above quantities have been recomputed using the $\mathrm{TB}+\mathrm{Q}$ theory in place of the simple TB theory, but the differences are not significant.

According to the modern understanding [23], the electronic contribution to the polarization can be equivalently expressed either in terms of a Berry phase (BP) computed from the Bloch functions, or in terms of the displacements of the Wannier centers (i.e., from the molecular dipole moments). These two approaches are compared for each of the three different computational schemes (TB, TB $+\mathrm{Q}$, and LDA) in Table V1. It can be seen that the bulk polarizations $P_{z}$ computed from the WF and BP approaches are in good agreement with each other $(\sim 5 \%)$ for all three schemes, indicating good internal consistency. (The small discrepancies can be traced mainly to incomplete $k$-point convergence.)

The advantage of the WF approach is that the decomposition into molecular dipole moments can give some insight into the microscopic origins of the dielectric prop- 
TABLE V. Spatial distribution of Wannier functions for the first three shells. Shown in the table are the list of the nearest neighboring molecules, their distance to home unit cell, the contribution to probability from each molecule, and the accumulated probability up to the current molecule.

\begin{tabular}{cccc}
\hline \hline Neighbor & Radius (a.u.) & Probability & Accum. Prob. \\
\hline 0 & 0.0000 & 0.84337 & 0.84337 \\
\hline 1 & 3.4342 & 0.00764 & 0.85101 \\
2 & 3.4342 & 0.00764 & 0.85865 \\
3 & 3.4342 & 0.01294 & 0.87159 \\
4 & 3.4342 & 0.01294 & 0.88453 \\
5 & 3.4342 & 0.01898 & 0.90352 \\
6 & 3.4342 & 0.00648 & 0.91000 \\
\hline 7 & 3.5162 & 0.00984 & 0.91984 \\
8 & 3.5162 & 0.00984 & 0.92969 \\
9 & 3.5162 & 0.01098 & 0.94067 \\
10 & 3.5162 & 0.01098 & 0.95165 \\
11 & 3.5162 & 0.00777 & 0.95942 \\
12 & 3.5162 & 0.00777 & 0.96719 \\
\hline \hline
\end{tabular}

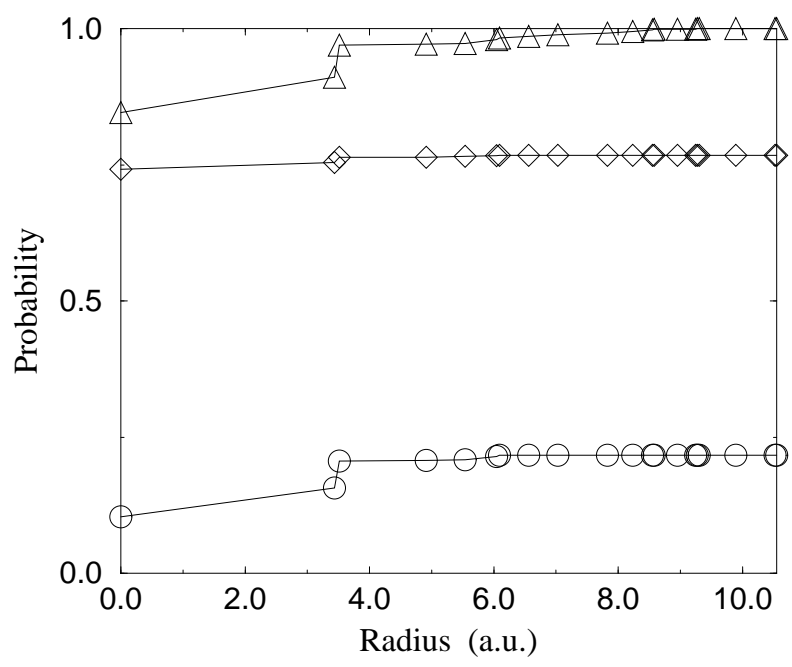

FIG. 13. The spatial distribution and decomposition of Wannier functions. $\bigcirc$ is the contribution to probability from $p$-orbitals, $\diamond$ is the contribution from the $s$-orbitals, and $(\triangle)$ is the sum of the $s$ and $p$ contributions.
TABLE VI. Comparison of dipole moments and bulk polarization calculated by TB, TB+Q and LDA. Because of the symmetry of $C m c 2_{1}, d_{1 x}=d_{2 x}=0, d_{1 y}=-d_{2 y}\left(\right.$ so $\left.P_{y}=0\right)$, and $d_{1 z}=d_{2 z}$. The results from the Wannier-function analysis are given in the first three rows, while the bulk polarization from Berry-phase calculation appears in the last row. Atomic units are used.

\begin{tabular}{lccc}
\hline \hline & TB & TB $+\mathrm{Q}$ & LDA \\
\hline$d_{1 y}$ & -0.03326 & -0.03759 & -0.06297 \\
$d_{1 z}$ & 0.01252 & 0.00894 & -0.00412 \\
$P_{z}$ & 0.000422 & 0.000301 & -0.000139 \\
$P_{z}(\mathrm{BP})$ & 0.000436 & 0.000315 & -0.000143 \\
\hline \hline
\end{tabular}

erties. As in the LDA calculations, we find larger $d_{y}$ components and smaller $d_{z}$ components, with a pattern of signs determined by symmetry requirements. The interpretation of the vibron infrared activities in terms of this picture has already been discussed in Sec. IV.

Unfortunately, the level of agreement between the TB and LDA results for the molecular dipole moments is somewhat disappointing. We find a $\mathrm{TB} d_{y}$ value that has the right sign, and the correct order of magnitude, relative to the LDA value, but the actual values differ by a factor of about two. The TB $d_{z}$ value even has the wrong sign, but this is related to the fact that the LDA $d_{z}$ value happens to come out very small ( $\sim 10$ times smaller than for $d_{y}$; see also Fig. 6). Thus, it is not surprising that the relative TB error in $d_{z}$ is large, even though the absolute TB error is actually smaller for $d_{z}$ than for $d_{y}$. Because the simple TB theory does not include any charge self-consistency, it was hoped that the extension to the $\mathrm{TB}+\mathrm{Q}$ theory might improve the results by incorporating a leading (quadrupolar) Coulomb contribution. Table V1 shows that the changes from $\mathrm{TB}$ to $\mathrm{TB}+\mathrm{Q}$ are in the right direction, and there is some improvement in the $d_{z}$ (and therefore $P_{z}$ ) values, but the $d_{y}$ discrepancy is hardly affected.

Thus, while the TB theory gives a picture that is qualitatively correct, it is clear that there is room for improvement. Possible avenues for future investigation may be to consider nonorthonormal TB basis sets, to parameterize and include off-diagonal matrix elements of the position operators between TB basis functions, to include $s^{*}$ or $d$ orbitals in the TB basis, or to include other Coulomb contributions (e.g., a self-consistent inclusion of the field arising from the induced dipoles).

Finally, we calculated the shell-by-shell spatial decomposition of the molecular dipole moments in the TB scheme. The results appear in Fig. 14. (By symmetry, all $d_{x}$ contributions are identically zero.) Comparing Fig. 14 with Fig. 13, one sees that although the contribution up to the first neighbor shell is $91 \%$ for the density, it is only $62 \%$ and $60 \%$ for $d_{y}$ and $d_{z}$, respectively. Up to the second shell, the contribution is $97 \%$ for the density and $95 \%$ for $d_{y}$, but only $67 \%$ for $d_{z}$. (Of course, $d_{z}$ is the only component that survives in the summation giving 


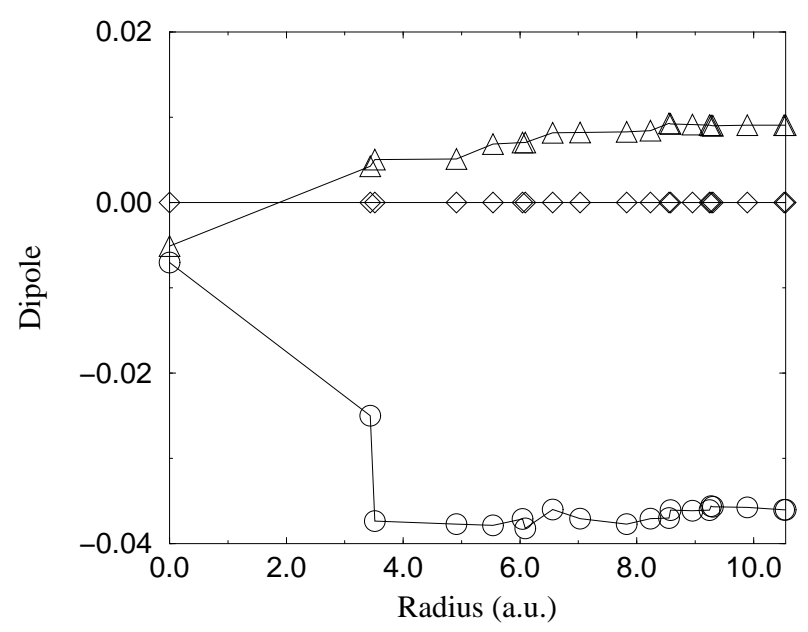

FIG. 14. Shell-by-shell contribution to dipole. The horizontal line $(\diamond)$ is the dipole along the $x$-direction. $\bigcirc$ and $\triangle$ indicate the contributions to dipole along $y$ and $z$ directions, respectively. The dipole is in a.u.

the bulk polarization.) Thus, the dipoles are found to be very delocalized, spanning over quite a few neighboring molecules, in agreement with the LDA results.

To summarize this section, we have demonstrated that an empirical TB framework allows for a very useful qualitative (and often semiquantitative) analysis of the WFs in systems such as the $\mathrm{H}_{2}$ phases under study here. It is typical of the empirical TB approach that one cannot insist on quantitative accuracy at the level of firstprinciples schemes. However, the TB approximation has proven enormously useful over the years because of its simplicity, transparency, and ease of application. These features often allow for insightful modeling of simple systems, or for efficient calculations of large and complex systems where ab-initio schemes would not be practical. For example, one could easily use the present scheme for a computationally efficient analysis of the local dielectric structure of more complex $\mathrm{H}_{2}$ crystal structures [10] or of supercell realizations of disordered $\mathrm{H}_{2}$ systems [50]. We expect that the coupling of Wannier and TB approaches will prove to be a useful strategy in a wide variety of other materials systems as well.

\section{DISCUSSION}

\section{A. Wannier functions and the Clausius-Mossotti approximation}

As discussed by Harrison 49, WFs provide a rigorous formulation of the "extended bond orbitals" which appear in tight-binding models. It should be noted that orbitals more localized than WFs can be constructed by removing the orthogonality constraint, which gives rise to the long-range tails; the resulting orbitals correspond to the "bond orbitals" in Harrison's picture. However, the straightforward connection to the polarization is then lost, since $\mathbf{P}_{\text {mac }}$ is no longer simply the sum of the dipole moments of those orbitals, and additional cross-terms connecting orbitals in different cells appear. Therefore, as far as dielectric properties are concerned, the "maxloc" WFs seem to provided the most localized description of the electronic structure. In particular, the spatial extent of their dipole moments (Figs. 6 and 14 ) provides a natural length scale to compare with the intermolecular distance in order to assess the validity of the ClausiusMossotti approximation of nonoverlapping dipoles.

\section{B. Identifying "molecules" in the dense solid}

There is a vast literature dealing with useful ways of identifying individual "atoms" inside a molecule, or individual "molecules" in a dense medium (see Refs. 5456 and references cited therein). In this paper we have advocated using "maxloc" WFs as a useful computational definition of " $\mathrm{H}_{2}$ molecules" in the insulating molecular solid (similarly, in Refs. 42,44] WFs were used for defining "water molecules" in liquid water). In spite of having some counterintuitive features (becoming larger under pressure) and some conceptual limitations (being defined only in the independent-electron framework; not being totally unique, since they depend on the measure of localization), they have the following important conceptual advantages. (i) When the Clausius-Mossotti approximation breaks down - which is when the ambiguity in identifying individual "molecules" appears - the sum of the dipoles of the Wannier molecules still gives the bulk polarization exactly (Eq. 5). By contrast, any definition of "molecules" based upon a direct partition of the bulk electronic charge density necessarily yields an incorrect result, since away from the Clausius-Mossotti limit the information about the bulk $\mathbf{P}_{\text {mac }}$ is not in $\rho(\mathbf{r})$ [23]. For example, in Ref. [56] it was found that different schemes for partitioning the charge density yield very different molecular dipoles in liquid water. We would expect the situation with such approaches to be even more severe in the case of solid hydrogen, since the dipoles are smaller and originate mostly in the Wannier tails. (ii) At high densities the WFs interpenetrate one another, so that the charge density at a given point is a sum of contributions from different molecules. Thus, effects related to molecular overlap are naturally discussed in the Wannier representation. 


\section{Intramolecular versus intermolecular charge transfer}

There has been some debate about whether the strong vibron IR activity in compressed solid hydrogen is due mainly to intramolecular or intermolecular charge transfer [17,28,30]. As a result of the ambiguity in defining "molecules" in the solid, the question is to some extent ill-posed. Of course, there is no charge transfer between "Wannier molecules", since their charge is fixed. However, a heuristic argument can be attempted: the fraction of the vibron-induced molecular effective charge originating in the central part of the WF can be viewed as the "intramolecular" contribution (polarization of the molecular bond), whereas the contributions from the orthogonality tails in the overlap regions are of "intermolecular" origin. As the pressure increases, the "intramolecular" part of the WF becomes smaller and contains less charge (and presumably becomes less polarizable), whereas the opposite happens to the outer corona. The results of Secs. V and VII suggest that as far as polarization-and hence IR activity-are concerned, at megabar pressures the "intermolecular" contribution associated with the outer corona is dominant.

\section{Summary}

Using a method for computing well-localized Wannier functions [3], we have presented a "chemical-like" localized picture of the electronic structure of solid molecular hydrogen, and used it to investigate the dielectric properties of the compressed system. This approach is particularly well-suited for studying the effects of molecular overlap, which become increasingly more important at high pressures. We found the somewhat surprising result that already at moderate pressures the orthogonality tails of the WFs in the overlap regions give rise to most of the induced dipole moments on the "Wannier molecules"; this clearly indicates a breakdown of the Clausius-Mossotti approximation. Under those circumstances the electric polarization cannot be extracted from the electronic charge density in the unit cell, and the Berry-phase/Wannier-function theory [23] must be used instead. The present approach clarifies the origin of the strong vibron IR activity in phase III and identifies the dominant mechanism: even though the permanent dipoles of the molecules in our prototype structures are too small to account for the vibron oscillator strength, the vibron-induced dipole fluctuations are of the right order of magnitude in $C m c 2_{1}$, and actually too large in $C 2 / \mathrm{m}$. In other words, in the strongly compressed solid the dynamical contribution to the vibron effective charge dominates the static one. This conclusion seems to be supported by the fact that, even though several libron modes have been identified in phase III [35, 36], no strong libron IR activity has been reported. If the molecules had any significant spontaneous polarization, it should manifest itself in the librational IR absorption. Thus, we see that well-localized Wannier functions provide a useful definition of " $\mathrm{H}_{2}$ molecules" in the dense solid, which can be used to gain important insight into the microscopic mechanisms of its dielectric response.

\section{ACKNOWLEDGMENTS}

I.S. and R.M.M. acknowledge financial support from DOE Grant No. DEFG02-96-ER45439, X.Z. and D.V. from NSF Grant DMR-9981193. I.S. acknowledges financial support from FCT (Portugal).

[1] G. H. Wannier, Phys. Rev. 52, 191 (1937).

[2] E. I. Blount, Solid State Phys. 13, 305 (1962).

[3] N. Marzari and D. Vanderbilt, Phys. Rev. B 56, 12847 (1997).

[4] Interestingly, one of the earliest attempts at constructing Wannier functions in an ab initio fashion was precisely for solid hydrogen, but in a metallic and atomic structure: W. Andreoni, Phys. Rev. B 14, 4247 (1976).

[5] H. K. Mao and R. J. Hemley, Rev. Mod. Phys. 66, 671 (1994).

[6] K. A. Johnson and N. W. Ashcroft, Nature (London) 403, 632 (2000).

[7] M. Städele and R. M. Martin, Phys. Rev. Lett. 84, 6070 (2000).

[8] C. Narayana, H. Luo, and A. L. Ruoff, Nature (London) 393, 46 (1998).

[9] E. Kaxiras and J. Broughton, Europhys. Lett. 17, 151 (1992).

[10] J. Kohanoff, S. Scandolo, S. Gironcoli, and E. Tosatti, Phys. Rev. Lett. 83, 4097 (1999).

[11] B. Edwards and N. W. Ashcroft, Nature (London) 388, 652 (1997).

[12] A class of orthorhombic structures, with the Cmca symmetry, was also found to be energetically competitive [57.13. However, these structures are metallic, in contradiction with experiment.

[13] H. Kitamura, S. Tsuneyuki, T. Ogitsu, and T. Miyake, Nature (London) 404, 259 (2000).

[14] R. Zallen, R. M. Martin, and V. Natoli, Phys. Rev. B 49, 7032 (1994).

[15] L. Cui, N. H. Chen, and I. F. Silvera, Phys. Rev. B 51, 14987 (1995); ibid 57, 656 (1998).

[16] M. Hanfland, R. J. Hemley, and H. K. Mao, Phys. Rev. Lett. 70, 3760 (1993).

[17] R. J. Hemley, I. I. Mazin, A. F. Goncharov, and H. K. Mao, Europhys. Lett. 37, 403 (1997).

[18] P. Loubeyre et al., Nature (London) 383, 702 (1996).

[19] S. Califano and V. Schettino, Lattice Dynamics of Molecular Crystals (Springer-Verlag, Berlin, 1981). 
[20] R. W. Munn, Mol. Phys. 64, 1 (1988), and references cited therein.

[21] B. L. Smith, The Inert Gases - Model Systems for Science (Wykeham Publications, London, 1971), p. 118.

[22] R. M. Martin, Phys. Rev. B 9, 1998 (1974).

[23] R. D. King-Smith and D. Vanderbilt, Phys. Rev. B 47, 1651 (1993); D. Vanderbilt and R. D. King-Smith, Phys. Rev. B 48, 4442 (1993); R. Resta, Rev. Mod. Phys. 66, 899 (1994).

[24] For some molecular crystals, such as solid $\mathrm{I}_{2}$, overlap effects are known to be significant already at ambient pressure. See, for example, A. Pasternak, A. Anderson, and J. W. Leech, J. Phys. C 10, 3261 (1977).

[25] I. Souza and R. M. Martin, Phys. Rev. Lett. 81, 4452 (1998).

[26] The $\mathrm{H}_{2}$ molecules are modeled as point objects with a cylindrically symmetric quadrupole moment and polarizability, which become polarized in the quadrupolar electric field of the surrounding molecules. The values of the quadrupole moment and polarizability as a function of bond length are from Refs. 39] and 40] respectively.

[27] B Baranowski, Pol. J. Chem. 66, 1637 (1992).

[28] B Baranowski, Pol. J. Chem. 69, 981 (1995).

[29] I. I. Mazin, R. J. Hemley, A. F. Goncharov, M. Hanfland, and H. K. Mao, Phys. Rev. Lett. 78, 1066 (1997).

[30] R. J. Hemley, Z. G. Soos, M. Hanfland, and H. K. Mao, Nature (London), 369, 384 (1994); Z. G. Soos, J. H. Eggert, R. J. Hemley, M. Hanfland, and H. K. Mao, Chem. Phys. 200, 23 (1995); Z. G. Soos and D. Mukhopadhyay, Chem. Phys. Lett. 245, 194 (1995).

[31] O. Schnepp, J. Chem. Phys. 46, 3983 (1967).

[32] Since we are neglecting the small coupling between the vibron and the other lattice modes, we choose the normal coordinate to be the change in molecular bond length $r_{\text {bond }}(1)$ of molecule 1 from its equilibrium value: $u_{\nu}=$ $\delta r_{\text {bond }}(1)$.

[33] P. Ghosez, J.-P. Michenaud, and X. Gonze, Phys. Rev. B 58, 6224 (1998).

[34] In Ref. [33 Eq. 9 is discussed for an isolated diatomic molecule. In that case the effective charge vector is directed along the molecular axis, so that a scalar definition is adequate, since at most the signs of the static and dynamical terms may differ. The vectorial definition becomes relevant for the Wannier molecules in the solid: because there the symmetry is lower, the effective charge vectors can point along other directions, which in general also differ between the static and dynamical terms.

[35] A. F. Goncharov, R. J. Hemley, H. K. Mao, and J. Shu, Phys. Rev. Lett. 80, 101 (1998).

[36] R. J. Hemley, A. F. Goncharov, H. K. Mao, E. Karmon, and J. H. Eggert, J. Low Temp. Phys. 110, 75 (1998).

[37] P. P. Edwards and F. Hansel, Nature (London) 388, 621 (1997).

[38] The results in Ref. 17 are presented in terms of Szigeti effective charges, obtained, under the approximation of cubic symmetry, by multiplying the Born charges by $[\epsilon(\infty)+2] / 3[33$. We converted them back to Born charges using $\epsilon(\infty)=n^{2}=(2.7)^{2}$ [58]. Moreover, we multiplied the effective charges given in Ref. [17] by $\sqrt{3}$, in order to take into account the averaging over all direc- tions, due to the use of polycrystalline samples. Unlike the Szigeti charges, which are model-dependent quantities since they are related to local fields, the Born charges are macroscopic, model-independent, measurable quantities.

[39] J. D. Poll and L. Wolniewicz, J. Chem. Phys. 68, 3053 (1978).

[40] W. Kolos and L. Wolniewicz, J. Chem. Phys. 46, 1426 (1967).

[41] I. I. Mazin and R. E. Cohen, Phys. Rev. B 52, R8597 (1995).

[42] P. L. Silvestrelli and M. Parrinello, J. Chem. Phys. 111, 3572 (1999).

[43] H. Werner and W. Meyer, Mol. Phys. 31, 855 (1976).

[44] P. L. Silvestrelli and M. Parrinello, Phys. Rev. Lett. 82, 3308 (1999).

[45] C. A. ten Seldam and S. R. de Groot, Physica 18, 905 (1952).

[46] T. H. Keil, J. Chem. Phys. 46, 4404 (1967).

[47] R. Resta and S. Sorella, Phys. Rev. Lett. 82, 370 (1999).

[48] I. Souza, T. Wilkens, and R. M. Martin, Phys. Rev. B 62, 1666 (2000).

[49] W. A. Harrison, Electronic Structure and the Properties of Solids (Freeman, New York, 1980).

[50] H. Chacham, L.M. Falicov, and B. Koiller, Phys. Rev. B 50, 7195 (1994).

[51] D. Sánchez-Portal, I. Souza, and R. M. Martin, Proceedings of the 2000 Aspen Winter Conference on Fundamental Physics of Ferroelectrics, R.E. Cohen, ed. (AIP, Woodbury, New York, 2000), in press.

[52] J. Bennetto and D. Vanderbilt, Phys. Rev. B. 53, 15417 (1996).

[53] H. Chacham, S. G. Louie, Phys. Rev. Lett 66, 64 (1991).

[54] R. Bader, Atoms in Molecules. A Quantum Theory (Oxford University Press, Oxford, 1990).

[55] L. Delle Site, A. Alavi, and R. M. Lynden-Bell, Mol. Phys. 96, 1683 (1999).

[56] E. R. Batista, S. S. Xantheas, and H. Jónsson, J. Chem. Phys. 111, 6011 (1999).

[57] B. Edwards, N. W. Ashcroft, and T. Lenosky, Europhys. Lett. 34, 519 (1996).

[58] R. J. Hemley, H. K. Mao, A. F. Goncharov, M. Hanfland, and V. Struzhkin, Phys. Rev. Lett. 76, 1667 (1996). 